\title{
Influence of weak-layer heterogeneity on snow slab avalanche release: application to the evaluation of avalanche release depths
}

\author{
Johan GAUME, Guillaume CHAMBON, Nicolas ECKERT, Mohamed NAAIM \\ Irstea, UR ETGR, Saint-Martin-d'Hères, France \\ E-mail: johan.gaume@gmail.com
}

\begin{abstract}
The evaluation of avalanche release depths constitutes a great challenge for risk assessment in mountainous areas. This study focuses on slab avalanches, which generally result from the rupture of a weak layer underlying a cohesive slab. We use the finite-element code Cast3M to build a mechanical model of the slab/weak-layer system, taking into account two key ingredients for the description of avalanche release: weak-layer heterogeneity and stress redistribution via slab elasticity. The system is loaded by increasing the slope angle until rupture. We first examine the cases of one single and two interacting weak spots in the weak layer, in order to validate the model. We then study the case of heterogeneous weak layers represented through Gaussian distributions of the cohesion with a spherical spatial covariance. Several simulations for different realizations of weak-layer heterogeneity are carried out and the influence of slab depth and heterogeneity correlation length on avalanche release angle distributions is analyzed. We show, in particular, a heterogeneity smoothing effect caused by slab elasticity. Finally, this mechanically based probabilistic model is coupled with extreme snowfall distributions. A sensitivity analysis of the predicted distributions enables us to determine the values of mechanical parameters that provide the best fit to field data.
\end{abstract}

\section{INTRODUCTION}

The evaluation of avalanche release depths is of great importance for all applications related to hazard mapping or zoning. In particular, avalanche release depths represent a crucial input ingredient for dynamical runout models (Barbolini and others, 2000; Naaim and others, 2003) and are required for implementing statistical-dynamical simulations (Meunier and Ancey, 2004; Eckert and others, 2010). It has been shown in various studies (Hutter, 1996; Bartelt and others, 1999; Jamieson and others, 2008) that the outputs of these models in terms of runout distances and impact forces are strongly dependent on the release mass, as well as on other terms, such as friction, deposition and erosion.

It is commonly accepted that dry-snow slab avalanches are initiated by a shear failure in a weak snow layer (or at a weak interface) followed by tensile crown failure of the overlying slab (McClung, 1979; Schweizer and others, 2003). The shear failure is caused by a local loss of cohesion inside the weak layer, that may be due to (1) a localized surface loading, such as skiers or explosives (artificial release), (2) uniform loading due to a new snowfall (natural release) or (3) changes in the snowpack properties due to weather changes (natural release).

Most of the existing avalanche release models are based on the assumption first made by Palmer and Rice (1973) for an overconsolidated clay and then taken up for snow by McClung (1979), that a weak spot or shear band, i.e. a zone of zero shear strength, pre-exists inside the weak layer. Fracture mechanics is then applied to study the conditions for shear-band propagation. Based on an energy budget at the tip of the band, rapid propagation occurs when a critical length is reached after a phase of slow strain-softening. Schweizer (1999) produced a complete review of these fracture mechanics models and gave critical length values as a function of snow characteristics. More recently, Chiaia and others (2008) showed that a simple stress failure criterion from equilibrium equations could also be sufficient to predict shear-band propagation with good accuracy. Nevertheless, as already noted by Schweizer (1999), the very concept of a weak spot is questionable since, even if we can imagine how local weak zones could appear (e.g. around rocks, where the snow depth is reduced and thus the temperature gradient is increased), it is probably too simplistic, in general, to represent the complex heterogeneity of weak-layer mechanical properties.

Several studies (Conway and Abrahamson, 1988; Jamieson and Johnston, 2001; Birkeland and others, 2004; Kronholm, 2004; Schweizer and others, 2008; Bellaire and Schweizer, 2011) have shown that snow mechanical properties present considerable spatial variability. From field data, this variability is generally described as following Gaussian distributions with spatial correlations. Hence, the concept of weak spot may be replaced by mechanical models that take into account spatial stochastic processes to represent the heterogeneity. Such models would explain not only failure initiation in weak zones, but also fracture arrest in stronger zones (Schweizer, 1999). Recently, several studies have attempted to include this heterogeneity in mechanical models. These studies can be classified as follows, according to the numerical method used:

1. Fiber bundle models (FBM) are simple statistical fracture models that are well adapted for representing spatially heterogeneous systems, including possible time-dependent effects, such as sintering. Using this framework, Reiweger and others (2009) model the weak snow layer as a discrete set of parallel brittle-elastic fibers. Spatial variability is accounted for by assigning each fiber an initial strength taken within a Weibull distribution. Despite the simplicity of the model, these authors are able to quantitatively reproduce laboratory shearing experiments on homogeneous snow samples. However, complex stress redistribution effects from elasticity of the 
slab cannot be taken into account in such models, which are therefore unable to reproduce full-scale avalanche release.

2. Cellular-automata models (CAM) consist of a regular grid of cells, characterized by a state that can change over time and as a function of the state of neighboring cells. Fyffe and Zaiser (2004, 2007) and Kronholm and Birkeland (2005) applied such an approach to slab avalanche release with a heterogeneous shear strength of the weak layer represented by Weibull or Gaussian distributions. Using neighboring elements, their models account for stress redistribution between weak and strong regions. They also included mode II (shear) rupture of the weak layer with a strain-softening law, and mode I (tensile) rupture of the slab, as did Failletaz and others (2004). These studies demonstrated the influence of spatial variability characteristics (variance, nugget effect, etc.) on release depth distributions. Fyffe and Zaiser (2007) were also able to reproduce (under certain conditions) release depth distributions following power laws, as in field studies (Rosenthal and Elder, 2002; McClung, 2003; Failletaz and others, 2004). However, stress elastic redistribution effects are oversimplified in these models, and their applicability is limited to the case of a shear strength correlation length lower than the slab depth.

3. Finite-element models (FEM) rely on the resolution of the complete mechanical equations of the problem. One of the main advantages of FEM compared to CAM is that they are able to capture large-scale stress redistribution effects due to elasticity. FEM has been successfully applied to modeling the mechanical response of sandwich specimens, including weak-snow layers with homogeneous properties (e.g. Bader and Salm, 1990; Stoffel, 2005; Mahajan and Joshi, 2008; Mahajan and others, 2010). However, only a few studies coupled FEM with a stochastic representation of the spatial variability. Recently, such a model was introduced by Griffiths and Fenton (2004) to study soil stability. To our knowledge, the same type of approach has not yet been undertaken for snow.

In addition, some recent studies (Johnson and others, 2004; Van Herwijnen and Heierli, 2009) relying on field data show that the shear failure of the weak layer tends to be systematically accompanied by a normal collapse. These authors argued that slope-normal and slope-parallel displacements occur simultaneously during release. Anticrack analytical models have been developed and have proved capable of reproducing these data (Heierli and Zaiser, 2008; Heierli and others, 2008). However, the issue of the influence of normal collapse on avalanche release is still a matter of debate. Some authors (Jamieson and Schweizer, 2000; Johnson and others, 2004) suggest that the simultaneous occurrence of weak-layer collapse and shear failure may facilitate fracture propagation due to bending effects. McClung (2011), however, showed that a model that does not account for slope-normal failure can reasonably reproduce critical length measurements obtained in field saw-cut tests. Hence, he argued that the slope-parallel propagation is very little influenced by the interaction between slopenormal displacement and stress. In the present study, the effect of normal collapse is not considered.
Here we use a finite-element method to build a mechanically based probabilistic model of the slab/weaklayer system, taking into account the two key ingredients: the redistribution effects by elasticity of the slab and the heterogeneity of weak-layer mechanical properties. The objective of this study is the evaluation of avalanche release depth statistical distributions, distributions that could be later coupled to propagation models for hazard mapping and zoning. In Sections 2 and 3, we present the system and validate the model using the well-known weak-spot case. This validation is also used to highlight a characteristic length of the system associated with the elastic redistribution of stresses. In Section 4, results obtained with a realistic spatial heterogeneity based on field data are presented. We analyze, in particular, how slab stability depends on slab depth and on the spatial correlation length of weak-layer properties. Finally, in Section 5, the obtained release depth distributions are combined with snowfall extreme value distributions and compared with field data of avalanche crown depths.

\section{MECHANICALLY BASED PROBABILISTIC MODEL}

\subsection{Objectives}

As mentioned above, the objective of the model developed in this study is the evaluation of probability distributions of avalanche release depths, in particular in the context of absent or scarce data. More precisely, the aim is to produce a tool capable of predicting release depth distributions that are meaningful over relatively long timescales (typically several decades), and that could be used as input for hazard mapping procedures, such as statistical-dynamical approaches (Keylock and others, 1999; Eckert and others, 2008, 2010). Hence, the objective is not to develop a complete mechanical model of slab avalanche release accounting for all the complex processes at play. Both the geometry and the mechanical behavior of the system are greatly simplified, to reduce the number of poorly known parameters, while keeping the ingredients essential to describe the mechanics of slab release. Moreover, to be compared with field data, the predictions of this model then need to be coupled with a description of snowfall distributions, as shown by Gaume and others (2012). In the present paper, we focus only on the formulation and validation (numerical consistency) of the mechanical part of the model. In Section 5, however, the sensitivity of the predicted distributions (after coupling with snowfalls) to several mechanical parameters is presented.

\subsection{Formulation of the model}

The model is based on the finite-element code Cast3M (Verpeaux and others, 1988). The resolution procedure used ('Pasapas'; Charras and Di Paola, 2011) enables the consideration of nonlinear models with an implicit integration scheme based on the weighted-residuals method. The momentum conservation equations, including inertial terms, are solved under the small deformations hypothesis:

$$
\begin{aligned}
& \mathbf{M} \cdot \ddot{\boldsymbol{u}}+\mathbf{D} \cdot \dot{\boldsymbol{u}}+\operatorname{div}(\boldsymbol{\sigma})=\boldsymbol{F}, \\
& \boldsymbol{\varepsilon}=\frac{1}{2}\left[\operatorname{grad}(\boldsymbol{u})+\operatorname{grad}^{\mathrm{t}}(\boldsymbol{u})\right],
\end{aligned}
$$

with $\mathbf{M}$ the mass matrix, $\boldsymbol{u}$ the node displacement vector, $\boldsymbol{\sigma}$ the stress tensor, $\boldsymbol{\varepsilon}$ the deformation tensor, $\boldsymbol{F}$ the integrated force vector at nodes and $\mathbf{D}$ the damping matrix. In our study, the matrix $\mathbf{D}$ is taken as zero. 


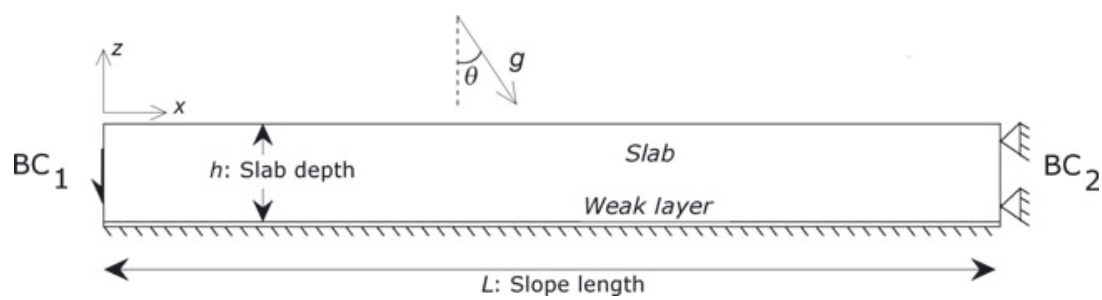

Fig. 1. Geometry of the system: a weak-layer interface under a cohesive slab of depth $h$.

The system considered is a two-dimensional (plane stress conditions) uniform slope inclined at an angle $\theta$, of length $L=50 \mathrm{~m}$ (Fig. 1). The $x$-axis is in the slope-parallel direction and the $z$-axis is orthogonal to the slope. The system consists of a slab of thickness $h$ overlying a weak layer, modeled as an interface of zero thickness. The mesh is composed of 100 elements in the slope-parallel direction, $x$, and six elements in direction $z$. We used quadrilateral elements for the slab (QUA4: four nodes with two degrees of freedom per node) and joint elements for the weak-layer interface (JOI2: four nodes with two degrees of freedom per node). We checked that the mesh resolution is fine enough not to influence the results presented (Section 3.1).

The boundary conditions applied to the slab are as follows: at the upper end of the slope (BC1) a shear stress, $\sigma_{x z}=-\rho g(z+h) \sin \theta$, is applied in order to avoid bending of the slab linked to finite-size effects; at the lower end (BC2), a nil displacement in the slope-parallel direction, $x$, is imposed. The upper surface of the slab is free and the base is subjected to an interface law, i.e. a law relating shear stress to tangential displacement, which represents the weak layer.

\subsection{Constitutive relationships}

Snow is a very complex material whose mechanical behavior is still not fully understood. In the present model, only the ingredients necessary to produce realistic instability of the system, namely strain-softening of the weak layer and elasticity of the slab, are taken into account. Table 1 summarizes the values of the different mechanical parameters used in this study.

\section{Weak layer}

Various studies (McClung, 1979; Föhn and others, 1998; McClung and Schweizer, 2006) have shown that weak snow layers behave as strain-softening (or quasi-brittle) materials. The softening is caused by the break of ice bridges at the microscopic scale. In existing mechanical models (McClung, 1979; Bažant and others, 2003; Fyffe and Zaiser, 2004, 2007; Mahajan and Joshi, 2008; Mahajan and others, 2010; Gaume and others, 2011), weak layers are generally characterized by a rupture displacement, $u_{\mathrm{p}}$, and a critical softening displacement, $\delta$. The pre-peak behavior is considered to be elastic, but stiffness values are very difficult to obtain, since these layers are generally very thin and unstable.

In the present study, the weak layer is modeled as a displacement-softening interface with a simple, linear piecewise relationship between shear stress, $\tau$, and tangential displacement, $u$ (Fig. 2). The value of the shear stress peak, $\tau_{\mathrm{p}}$, is governed by the Mohr-Coulomb criterion: $\tau_{\mathrm{p}}=c+\sigma_{\mathrm{n}} \tan \Phi$, with $c$ the weak-layer cohesion, $\sigma_{\mathrm{n}}$ the normal stress and $\Phi$ the friction angle. The friction angle is chosen as constant, $\Phi=30^{\circ}$ (De Montmollin, 1978; Van Herwijnen and Heierli, 2009), and the cohesion, $c$, is spatially heterogeneous, as described below. The tangential stiffness of the weak layer during the pre-peak phase is given by $k_{\mathrm{s}}=\tau_{\mathrm{p}} / u_{\mathrm{p}}$ (Fig. 2). After the peak, the shear stress decreases (shear softening), until reaching a residual value, $\tau_{\mathrm{r}}=\sigma_{\mathrm{n}} \tan \Phi$. This residual value corresponds to the situation where ice bridges are completely broken and only the friction between the slab and the underlying layer remains. Following McClung (1977), both the characteristic peak and softening displacement, $u_{\mathrm{p}}$ and $\delta$, are taken equal to $2 \mathrm{~mm}$ (Bažant and others, 2003; Fyffe and Zaiser, 2004, 2007). Hence, the displacement to reach the residual stress $u_{\mathrm{r}}=u_{\mathrm{p}}+\delta=4 \mathrm{~mm}$. Note that, according to recent studies (McClung, 2009, 2011), the softening displacement, $\delta$, under high loading rates tends to be smaller than the value assumed in this study, $0.1-0.3 \mathrm{~mm}$. However, as shown

Table 1. Mechanical parameters used in this study and typical ranges of variation

\begin{tabular}{|c|c|c|c|}
\hline Parameter & Typical value & Range & Source \\
\hline Slab density, $\rho$ & $250 \mathrm{~kg} \mathrm{~m}^{-3}$ & $100-300 \mathrm{~kg} \mathrm{~m}^{-3}$ & Schweizer (1999) \\
\hline Slab Young's modulus, $E$ & $1 \mathrm{MPa}$ & $0.2-12 \mathrm{MPa}$ & Schweizer (1999) \\
\hline Slab Poisson's ratio, $\nu$ & 0.2 & $0.1-0.4$ & Schweizer (1999) \\
\hline Slab viscosity, $\eta$ & $10^{8} \mathrm{~Pa} \mathrm{~s}$ & $0.2-5 \times 10^{8} \mathrm{Pas}$ & Mellor (1975); Camponovo and Schweizer (2001) \\
\hline Weak-layer cohesion, $c$ & $1 \mathrm{kPa}$ & $0.5-2.5 \mathrm{kPa}$ & $\begin{array}{c}\text { Föhn and others (1998); Jamieson and } \\
\text { Johnston (2001) }\end{array}$ \\
\hline Weak-layer friction angle, $\Phi$ & $30^{\circ}$ & $20-40^{\circ}$ & $\begin{array}{c}\text { De Montmollin (1978); Van Herwijnen and } \\
\text { Heierli (2009) }\end{array}$ \\
\hline Weak-layer displacement to failure, $u_{\mathrm{p}}$ & $2 \mathrm{~mm}$ & $1-10 \mathrm{~mm}$ & McClung (1977) \\
\hline Weak-layer residual displacement, $u_{\mathrm{r}}$ & $4 \mathrm{~mm}$ & $1-10 \mathrm{~mm}$ & McClung (1977) \\
\hline Correlation length, $\epsilon$, of weak-layer cohesion variations & $0.5-40 \mathrm{~m}$ & $0.5-10 \mathrm{~m}$ & Schweizer and others (2008) \\
\hline Coefficient of variation, $C_{V}=\sigma_{c} /\langle c\rangle$ & $30 \%$ & $15-50 \%$ & Schweizer and others (2008) \\
\hline
\end{tabular}




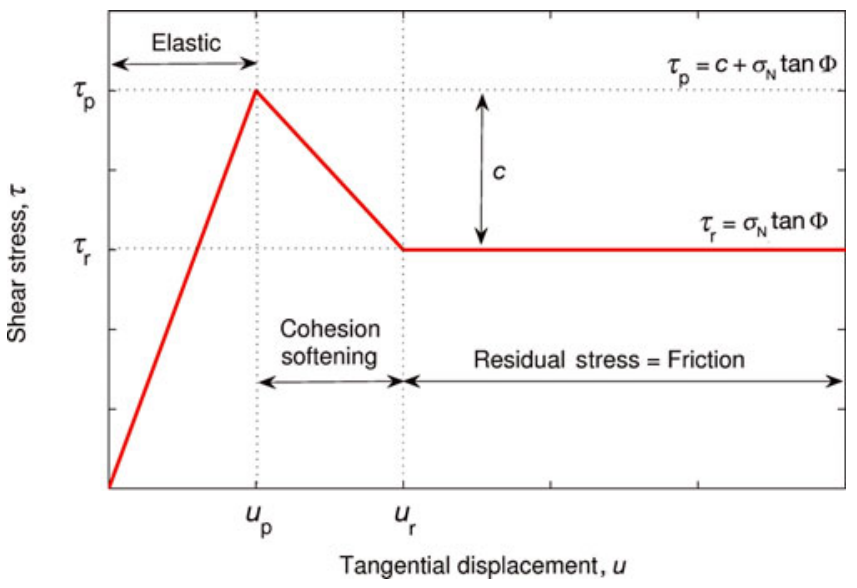

Fig. 2. Weak-layer constitutive law.

below, the precise value of this parameter does not influence the outcomes of our model.

\section{Slab}

At high loading rates, such as those characteristic of slab avalanche release, laboratory experiments (Mellor, 1975; McClung, 1977; Narita, 1980; Navarre and others, 1992; Schweizer, 1998) and field measurements (Roch, 1966; De Montmollin, 1978; Jamieson and Johnston, 1990; Föhn and others, 1998; McClung and Schweizer, 2006) have shown that cohesive snow behaves as a brittle-elastic material. In the present study (as explained in Section 2.6) the possible tensile rupture of the slab is not directly modeled. Therefore, the mechanical behavior of the slab is modeled by an isotropic elastic law:

$$
\varepsilon=\frac{1}{E} \sigma-\frac{\nu}{E}(\operatorname{tr}(\sigma)-\sigma),
$$

where $E$ is the Young's modulus and $\nu$ the Poisson's ratio.
The following values have been used: $E=1 \mathrm{MPa}, \nu=0.2$ and $\rho=250 \mathrm{~kg} \mathrm{~m}^{-3}$ for the slab density. In addition, in order to stabilize the computations, it has been necessary to slightly enrich the above constitutive law by the addition of a viscous term. All viscosity values within the range $10^{4}-$ $10^{9} \mathrm{Pas}$ have been found to yield satisfactory results. We retain the value $\eta=10^{8} \mathrm{~Pa}$ s, which is in agreement with real snow viscosity measurements (Mellor, 1975; Camponovo and Schweizer, 2001; Schweizer, 1999).

\subsection{Spatial heterogeneity}

The spatial heterogeneity of the weak layer is modeled through a stochastic distribution of cohesion, c. Following Jamieson and Johnston (2001) and Kronholm and Birkeland (2005), we consider a Gaussian distribution of average $\langle c\rangle$ and standard deviation $\sigma_{C}$, with a spherical covariance function $\mathcal{C}(d)$

$$
\mathcal{C}(d)=\sigma_{c}^{2}\left[1-\frac{3}{2} \frac{d}{\epsilon}+\frac{1}{2}\left(\frac{d}{\epsilon}\right)^{3}\right] \mathcal{I}_{[0, \epsilon]}(d),
$$

where $d$ is the distance between two points, $\epsilon$ is the spatial correlation length and the $\mathcal{I}_{\mathcal{A}}(d)$ function is 1 if $d \in \mathcal{A}, 0$ otherwise. The correlation length, $\epsilon$, represents the distance over which the cohesion values are significantly correlated. Note that in the present model, no nugget effect is considered (i.e. $\mathcal{C}(d) \rightarrow 0$ when $d \rightarrow 0$ ). The effect of the nugget on avalanche size has been investigated by Kronholm and Birkeland (2005) using a CAM.

Figure 3 shows examples of cohesion field realizations with different values of the correlation length, $\epsilon$. These fields were generated using the turning bands method (Chilès and Delfiner, 1999) and we checked that, with the used mesh size, the obtained empirical covariance functions are in good agreement with the predictions of Eqn (4) for all values of $\epsilon$ investigated. Existing studies are not conclusive on the typical correlation length scale (Jamieson and Johnston, 2001; Schweizer and others, 2008; Bellaire and Schweizer,
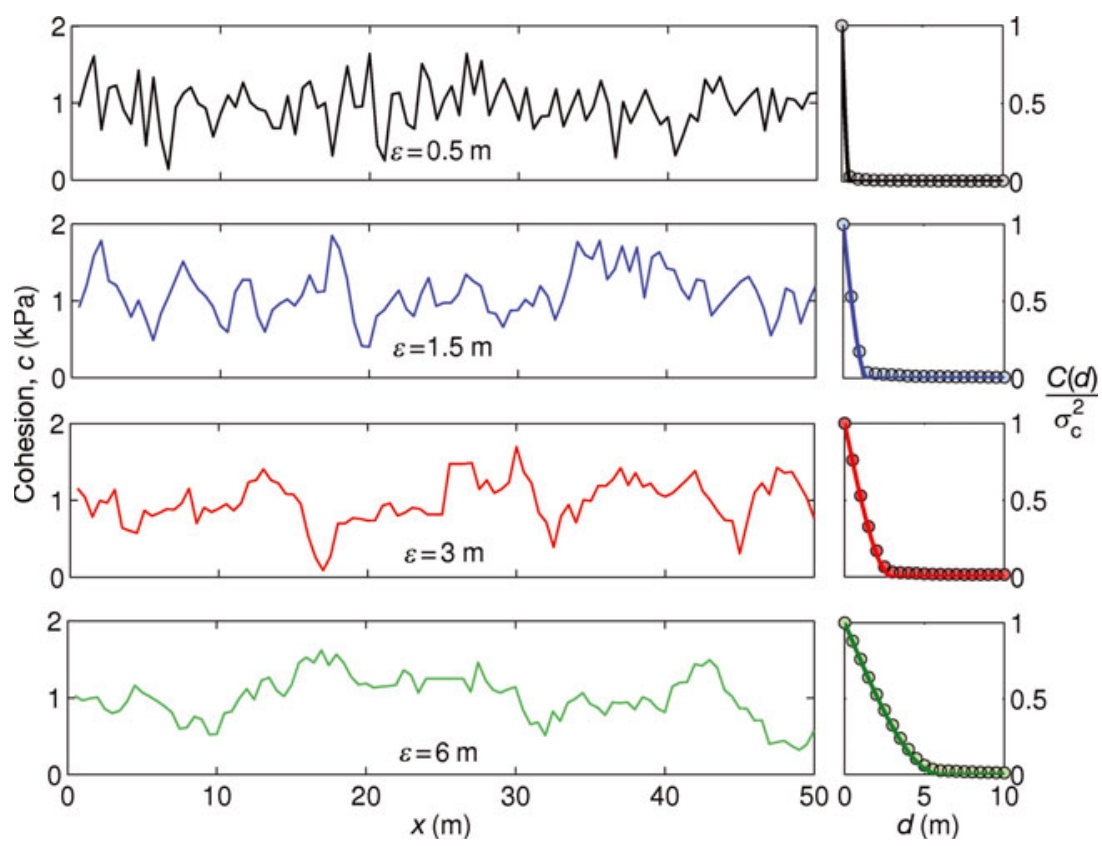

Fig. 3. Left: examples of the heterogeneity of the cohesion for different values of the correlation length, $\epsilon$. Right: comparison between the empirical normalized covariance function of the cohesion fields (computed from 100 independent realizations: circles) and the theoretical expression given by Eqn (4) (lines). 
2011) relevant for weak-snow layers. Schweizer and others (2008) recommended spacing snow pits at least $10 \mathrm{~m}$ apart in order to have independent results, thus suggesting that the correlation length, $\epsilon$, varies approximately within the $0.5-$ $10 \mathrm{~m}$ range. In our study, $\epsilon$ was varied between 0.5 and $40 \mathrm{~m}$ (the lower limit, $\epsilon=0.5 \mathrm{~m}$, being imposed by our mesh size), but, due to finite-size effects, only the results with $\epsilon \leq 10 \mathrm{~m}$ can be cross-compared (for $\epsilon>10 \mathrm{~m}$, the average cohesion, $\langle c\rangle$, begins to evolve with $\epsilon$ ). Lastly, the average cohesion, $\langle c\rangle$, was taken equal to $1 \mathrm{kPa}$ and cohesion standard deviation, $\sigma_{C}$, to $0.3 \mathrm{kPa}$.

\subsection{Loading}

Gravity is the only applied external force and the system is loaded by progressively increasing the slope angle, $\theta$, at constant slab depth until rupture. As will be shown, this loading procedure is equivalent to a progressive increase of the slab depth, $h$, at constant slope angle. The loading curve is shown in Figure 4. After an initial stage during which gravity is increased from 0 to $9.81 \mathrm{~m} \mathrm{~s}^{-2}$, the loading is applied in two phases (Fig. 4).

First, the slope angle is increased from 0 to $\theta_{1}=\Phi=30^{\circ}$ with a fast loading speed of $0.4^{\circ}$ per time-step, since no failure can occur during this stage $\left(\tau<\tau_{\mathrm{p}}\right)$. The loading speed is then reduced to $0.04^{\circ}$ per time-step until rupture occurs. We checked that, with this two-phase procedure, the chosen loading speed values do not influence the results presented. This simple loading is sufficient to study avalanche releases triggered by a progressive accumulation of snow. We emphasize that our model is not meant to account for the slow processes (snow metamorphism, viscous stress redistributions) active during the formation of the snowpack.

\subsection{Rupture mechanism}

Avalanche releases observed in our simulations are always induced by a local shear rupture inside the weak layer, which then propagates to both extremities of the slope. With reference to real avalanche releases, this corresponds to the case where the weak-layer heterogeneity is not sufficient to induce a tensile rupture within the slab, and slab rupture is thus triggered by morphological features, such as ridges, slope breaks, rocks, trees or other defects within the slab. We also performed simulations using a brittle-elastic constitutive law for the slab. These simulations showed that for a realistic tensile strength value $\left(\sigma_{\mathrm{t}}=2 \mathrm{kPa}\right.$; Mellor, 1975; Jamieson and Johnston, 1990; Sigrist, 2006), effectively no tensile failure occurred within the slab (the slab remained elastic) and the rupture mechanism was identical to that reported in this study. Note, however, that other sets of parameters may lead to tensile ruptures within the slab, which constitutes the subject of a further study (Gaume and others, 2011).

\subsection{Avalanche release criterion}

We consider that an avalanche occurs in our simulations when the following kinematic release criterion is met:

$$
\text { Release } \Leftrightarrow v_{x}^{i} \geq N \times{\overline{v_{x}}}^{i, m}=\frac{N}{m} \sum_{k=1}^{m} v_{x}^{i-k} \text {. }
$$

Hence, an avalanche is detected at time-step $i$ when the velocity, $v_{x}^{i}$, of any node of the weak layer is higher than $N$ times the average velocity recorded over $m$ previous timesteps, ${\overline{v_{x}}}^{i} m$. Values of $N=10$ and $m=10$ were chosen in order to ensure that the criterion is not sensitive to small

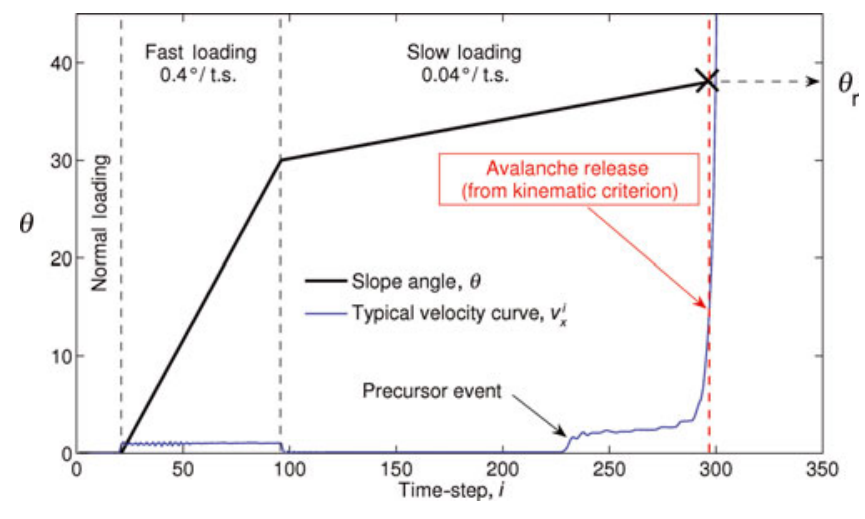

Fig. 4. Applied loading curve. The time-step (t.s.) is $0.1 \mathrm{~s}$. The blue curve represents a typical velocity evolution showing a marked precursor event.

velocity variations triggered by potential precursor events (Fig. 4), which could lead to incorrect release angle values.

\section{MECHANICAL VALIDATION}

In this section, the finite-element model presented above is validated against the classical case of release induced by a single weak spot. For this simple case, analytical solutions can be derived, following the approach of Chiaia and others (2008). The interaction between two weak spots is also considered, in order to illustrate the influence of an important characteristic length of the system that emerges from the analysis.

\subsection{A single weak spot}

\section{Analytical solution}

Here we follow the approach of Chiaia and others (2008), but considering a nonzero residual stress inside the weak spot due to friction (Fig. 2). Let us consider a weak spot of nil cohesion $(c=0)$, half-length $a$, inside a weak layer of homogeneous cohesion, $c=1 \mathrm{kPa}$, underlying a cohesive slab of depth $h$ (Fig. 5). The equilibrium equation in the slope-parallel direction integrated over the slab depth is

$$
\frac{\partial}{\partial x} \int_{-h}^{0} \sigma_{x x} \mathrm{~d} z-\tau=-\tau_{\mathrm{g}}
$$

with $\tau_{\mathrm{g}}=\rho g h \sin \theta$ the body weight shear stress, $\sigma_{x x}$ the normal stress in the slope-parallel direction and $\tau$ the shear stress in the weak layer. The shear stress, $\tau$, is related to the tangential displacement, $u$, according to the interface constitutive law (Fig. 2). Two cases have to be distinguished: $\theta<\Phi$ for which the shear stress, $\tau$, depends on the tangential displacement, $u$, both inside and outside the weak spot; and $\theta>\Phi$ for which the shear stress, $\tau$, depends on the tangential displacement, $u$, only outside the weak spot.

\section{Case 1: $\theta<\Phi$}

For $\theta<\Phi$, the shear stress is $\tau(x)=\tau_{\mathrm{p}} u / u_{\mathrm{p}}$ outside the weak spot $(|x|>a)$ and $\tau(x)=\tau_{\text {ws }}=\tau_{\mathrm{r}} u / u_{\mathrm{p}}$ inside the weak spot $(|x|<a)$. Equation (6) and the linear elastic behavior of the slab lead to

$$
\frac{\partial^{2} u}{\partial x^{2}}-\frac{u}{\Lambda(x)^{2}}=-\frac{\tau_{\mathrm{g}}}{E^{\prime} h^{\prime}}
$$




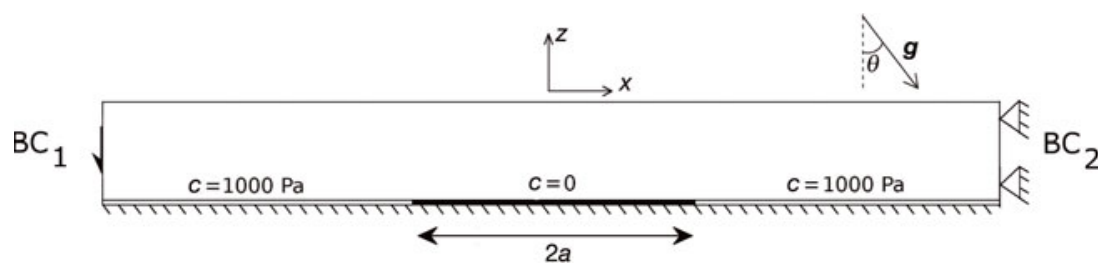

Fig. 5. Geometry of the system with one weak spot of half-length a with nil cohesion.

with $E^{\prime}=E /\left(1-\nu^{2}\right)$ (plane stress hypothesis) and

$$
\begin{gathered}
\Lambda(x)=\Lambda_{\mathrm{ws}}=\sqrt{\frac{E^{\prime} h u_{\mathrm{p}}}{\tau_{\mathrm{r}}}} \quad \text { for }|x| \leq a, \\
\Lambda(x)=\Lambda=\sqrt{\frac{E^{\prime} h u_{\mathrm{p}}}{\tau_{\mathrm{p}}}} \quad \text { for }|x|>a .
\end{gathered}
$$

Considering, in addition, the continuity of displacement and velocity at the interface between the weak layer and the weak spot, and the fact that the slope-parallel normal stress, $\sigma_{x x}$, vanishes far away from the weak spot, displacement and stress profiles can be determined by integrating Eqn (7).

Outside the weak spot $(|x|>a)$ :

$$
\left\{\begin{array}{l}
u(x)=\frac{\Lambda^{2} \tau_{\mathrm{g}}}{E^{\prime} h}\left(1-r \mathrm{e}^{\frac{-|x|-a}{\Lambda}}\right) \\
\tau(x)=\tau_{\mathrm{g}}\left(1-r \mathrm{e}^{-\frac{|x|-a}{\Lambda}}\right) .
\end{array}\right.
$$

Inside the weak spot $(|x| \leq a)$ :

$$
\left\{\begin{array}{l}
u(x)=\frac{\Lambda_{w s}^{2} \tau_{\mathrm{g}}}{E^{\prime} H}\left[1+r^{\prime}\left(\alpha \mathrm{e}^{x / \Lambda_{\mathrm{ws}}}+\beta \mathrm{e}^{-x / \Lambda_{\mathrm{ws}}}\right)\right] \\
\tau(x)=\tau_{\mathrm{g}}\left[1+r^{\prime}\left(\alpha \mathrm{e}^{x / \Lambda_{\mathrm{ws}}}+\beta \mathrm{e}^{-x / \Lambda_{\mathrm{ws}}}\right)\right]
\end{array}\right.
$$

with expressions for the constants $r, r^{\prime}, \alpha$ and $\beta$ given in Appendix A.

\section{Case 2: $\theta>\Phi$}

If $\theta>\Phi$, the shear stress inside the weak spot $(|x| \leq a)$ meets the frictional criterion and thus no longer depends on displacement, $u$ : $\tau(x)=\tau_{\mathrm{r}}=\sigma_{N} \tan \Phi$. Equation (6) then becomes

$$
\frac{\partial^{2} u}{\partial x^{2}}=\frac{\tau_{\mathrm{r}}-\tau_{\mathrm{g}}}{E^{\prime} h}
$$

for $|x| \leq a$ and Eqn (7) remains valid for $|x|>a$. Similarly to the previous case, the displacement and stress profiles can be determined.

Outside the weak spot $(|x|>a)$ :

$$
\left\{\begin{array}{l}
u(x)=\frac{\Lambda^{2} \tau_{\mathrm{g}}}{E^{\prime} h}\left(1-r_{2} \mathrm{e}^{-\frac{|x|-a}{\Lambda}}\right) \\
\tau(x)=\tau_{\mathrm{g}}\left(1-r_{2} \mathrm{e}^{-\frac{|x|-a}{\Lambda}}\right) .
\end{array}\right.
$$

Inside the weak spot $(|x| \leq a)$ :

$$
\left\{\begin{array}{l}
u(x)=\frac{\Lambda^{2} \tau_{\mathrm{g}}}{E^{\prime} h}\left\{\frac{1}{2} \frac{\Lambda}{\mathrm{a}} r_{2}\left[\left(\frac{x}{\Lambda}\right)^{2}-\left(\frac{\mathrm{a}}{\Lambda}\right)^{2}\right]+1-r_{2}\right\} \\
\tau(x)=\tau_{\mathrm{r},}
\end{array}\right.
$$

with the expression for the constant $r_{2}$ given in Appendix A.

We note that both the shear stress and the displacement present decreasing exponential profiles outside the weak spot (Eqns (10) and (13)). The characteristic length associated with these exponential decreases is the parameter $\Lambda$, which depends both on the slab and weak-layer characteristics (Eqn (9)). Far from the weak spot $(|x|-a \gg \Lambda)$, the shear stress tends to its body weight value, $\tau_{\mathrm{g}}$, and the displacement tends to $u=u_{\mathrm{p}} \tau_{\mathrm{g}} / \tau_{\mathrm{p}}$ (elastic behavior).

The shear band becomes unstable when the maximum stress, $\tau_{\max }$ at $|x|=a$ reaches $\tau_{\mathrm{p}}=c+\sigma_{N} \tan \Phi$. Using Eqn (13), the theoretical critical stress $\tau_{\mathrm{g}, \mathrm{s}}$ for weak spot propagation can thus be expressed as

$$
\tau_{\mathrm{g}, \mathrm{s}}=\frac{1}{1+\frac{\mathrm{a}}{\Lambda}}\left[c+\sigma_{N} \tan \Phi\left(1+\frac{\mathrm{a}}{\Lambda}\right)\right] .
$$

From this expression, the critical release angle, $\theta_{r, s}$, can then be derived using

$$
\tau_{\mathrm{g}, \mathrm{s}}=\rho g h \sin \theta_{\mathrm{r}, \mathrm{s}}
$$
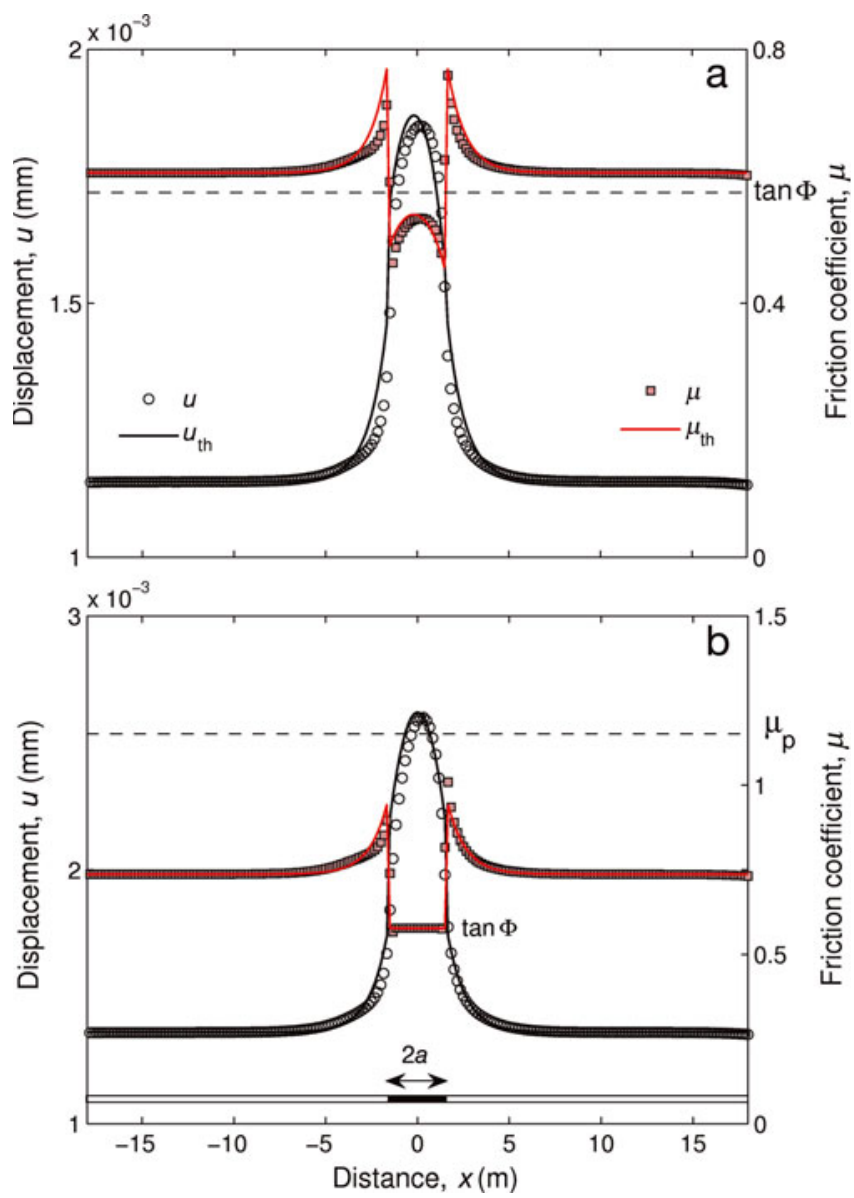

Fig. 6. Displacement $(u)$ and apparent friction coefficient $\left(\mu=\tau / \sigma_{\mathrm{n}}\right)$ profiles for a slab depth $h=1 \mathrm{~m}$ and a weak-spot halflength $\mathrm{a}=4.5 \mathrm{~m}$ for (a) the case $\theta<\Phi$ and (b) the case $\phi<\theta<\theta_{\mathrm{r}}$, where $\theta_{\mathrm{r}}$ is the release angle. Symbols: finite-element results ( $u$ and $\mu$ ). Solid curves: analytical model ( $u_{\mathrm{th}}$ and $\left.\mu_{\mathrm{th}}\right)$. 


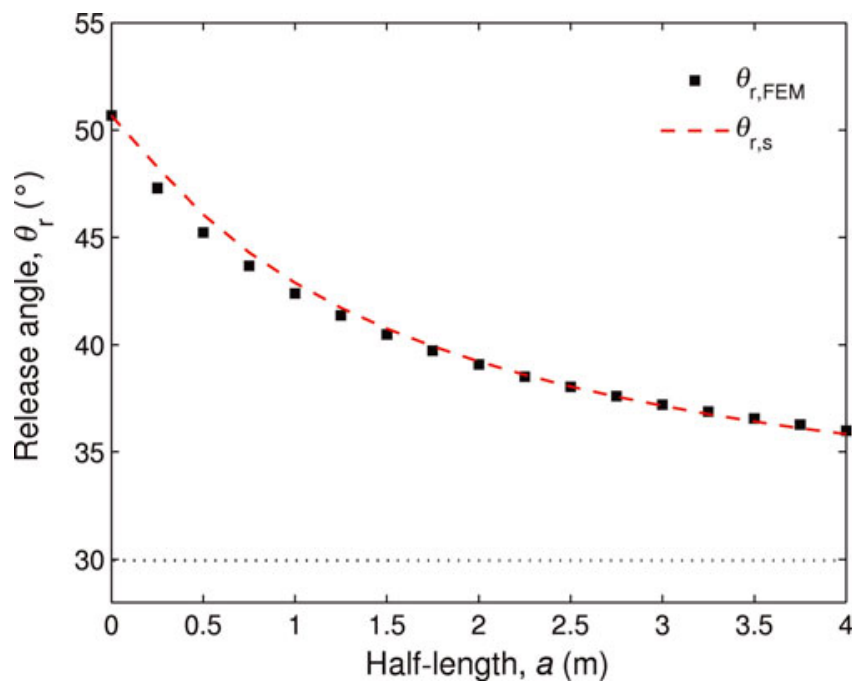

Fig. 7. Evolution of the release angle, $\theta_{r}$, as a function of the halflength, $a$, of the weak spot. Black squares: finite-element results. Red dashed curve: theoretical stress rupture threshold.

\section{Comparison with simulations}

As shown in Figure 6a and b, the overall agreement between theoretical predictions and FEM numerical results is very satisfactory, both for $\theta<\Phi$ and for $\theta>\Phi$. In particular, the stress concentration at the weak-spot tip and the exponential decrease of stress outside the weak spot are very well reproduced. Similarly, the displacement profiles, which present a maximum at the center of the weak spot, are also well captured. Note, however, that the numerical model indicates the existence of slight variations with $x$ of the normal stress, $\sigma_{\mathrm{n}}$, which are not accounted for in the theoretical analysis.

Figure 7 shows a comparison between the release angles obtained by the FEM calculations and those predicted by the stress rupture criterion (Eqns (15) and (16)). Here also, the agreement between the theory and numerical results is excellent for all values of weak-spot half-lengths. This agreement also holds for all tested values of slab depth, $h$. Globally, the results shown in Figures 6 and 7 constitute a validation of the various mechanical ingredients taken into account in our finite-element model. In particular, these results prove that the used mesh size is fine enough to account for cohesion heterogeneities with typical length scales (in this case, the weak-spot half-length) as small as $0.5 \mathrm{~m}$.

\subsection{Two weak spots}

In order to illustrate the influence of the characteristic length, $\Lambda$, introduced above, we conducted simulations to investigate the interaction between two weak spots of length a separated by a distance $d$ (Fig. 8). Different values of $d$ were simulated and the effect of this parameter on the release angle was examined. (Note that for $d=0$ the problem is the same as that presented previously with one weak spot of half-length a.)

Figure 9 shows the evolution of the release angle as a function of distance, $d$. Typical displacement profiles illustrating the behavior for different values of $d / \Lambda$ are also shown. If the distance between the weak spots is high compared with $\Lambda$ (typically for $d / \Lambda \gtrsim 10$ ), then the displacement profiles generated by the weak spots do not interact. The release thus occurs for the same angle as for the case of only one weak spot of total length $a$. If the distance, $d$, between weak spots decreases to values of the same order as $\Lambda$ (i.e. if $1<d / \Lambda \lesssim 10$ ), the displacement profile retains a bimodal shape but the two peaks progressively coalesce. As a consequence of this interaction, the release angle, $\theta_{r}$, progressively decreases as $d$ decreases. Empirically, the evolution of $\theta_{\mathrm{r}}$ for $d / \Lambda>1$ can be adjusted by an exponential function: $\theta_{\mathrm{r}}=\theta_{\infty}\left(1-\gamma \mathrm{e}^{-d /(k \Lambda)}\right)$. As expected, the values of $\theta_{\infty}$ and $\gamma$ depend on the slab depth, $h$, and weak-spot length, $\mathrm{a}\left(\theta_{\infty} \approx 39.1^{\circ}\right.$ and $\gamma \approx 0.17$ in the present case), but the constant, $k$, is independent of these parameters $(k \approx 3)$. Finally, if the distance between weak spots is less than the characteristic length $(d / \Lambda<1)$, the release angle increases as $d$ decreases and the displacement profile becomes unimodal. This indicates that, in this case, the slab does not 'feel the effect' of the cohesive zone between the two weak spots and only 'sees an equivalent weak spot' of length $L \approx 2 a+d$.

Hence, it appears that the interaction and progressive bridging between the two weak spots is primarily controlled by the characteristic length, $\Lambda$. Physically, this characteristic length represents the typical distance over which the stress redistribution induced by slab elasticity is felt. As illustrated in Figure 9, this stress redistribution actually amounts to smoothing out the effect of the structural heterogeneity of the weak layer as soon as the typical variations of this heterogeneity occur over distances less than $\Lambda$. Hence, $\Lambda$ can be viewed as a characteristic smoothing length associated with slab elasticity. More generally, it should be noted that this parameter $\Lambda$ appears to be the main length scale of the system. In particular, we checked that the softening length, $\delta$, involved in the quasi-brittle weak-layer constitutive law, has essentially no influence on the results as long as it remains much smaller than $\Lambda$.

\section{RESULTS: INFLUENCE OF WEAK-LAYER HETEROGENEITY}

\subsection{Simulation protocol}

We now consider the case of a spatially heterogeneous weak layer, as described in Section 2.4. We conducted simulations

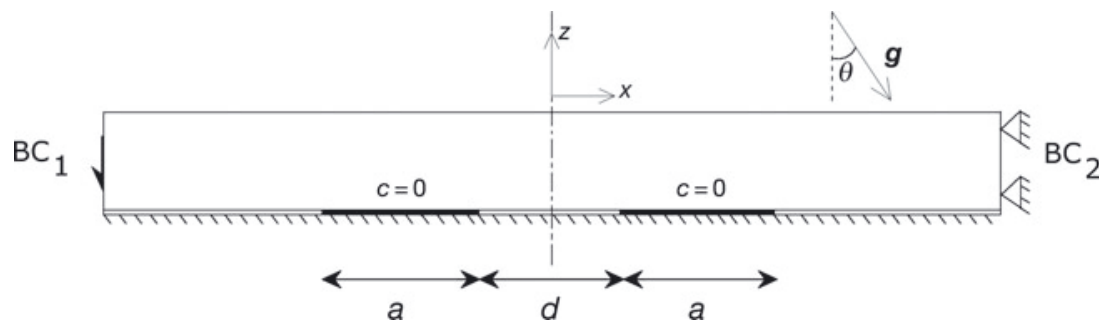

Fig. 8. Geometry of the system with two weak spots of length a separated by a distance $d$. 


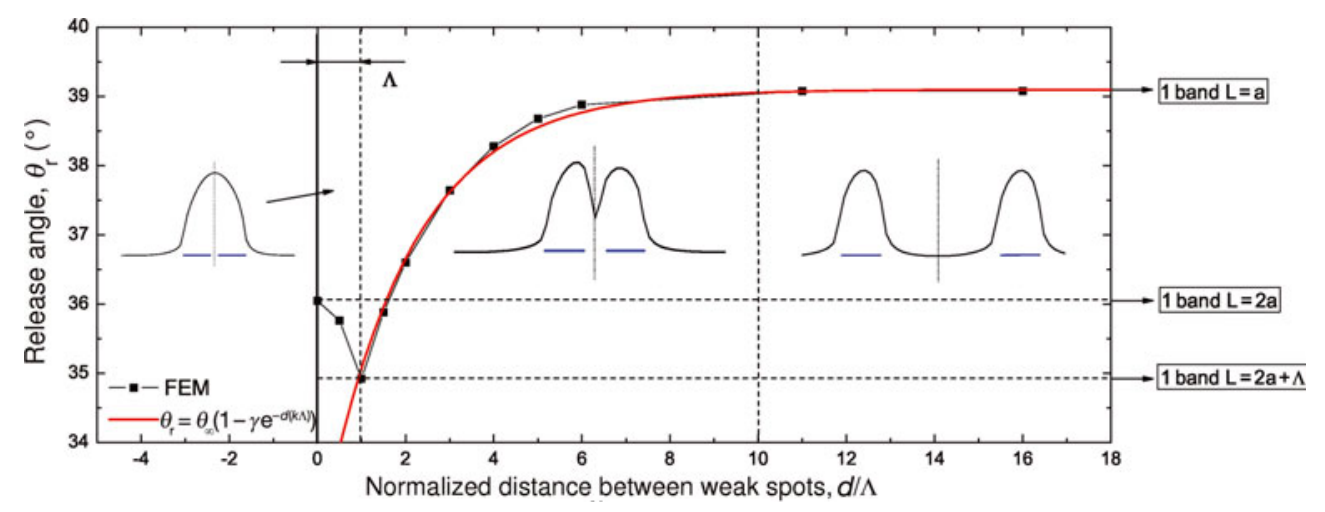

Fig. 9. Evolution of the release angle, $\theta_{\mathrm{r}}$, with the inter-distance between the two weak spots, $d$ (normalized by the characteristic length, $\Lambda$ ), for weak-spot lengths $a=4.5 \mathrm{~m}$. The black dots are the results of the finite-element model. The red curve is an exponential adjustment for $d / \Lambda>1$. Three different zones are distinguished: $d<\Lambda, \Lambda<d \lesssim 10 \Lambda$ and $d \gtrsim 10 \Lambda$, and the typical displacement profiles, recorded a few time-steps before release, in each of these zones are represented.
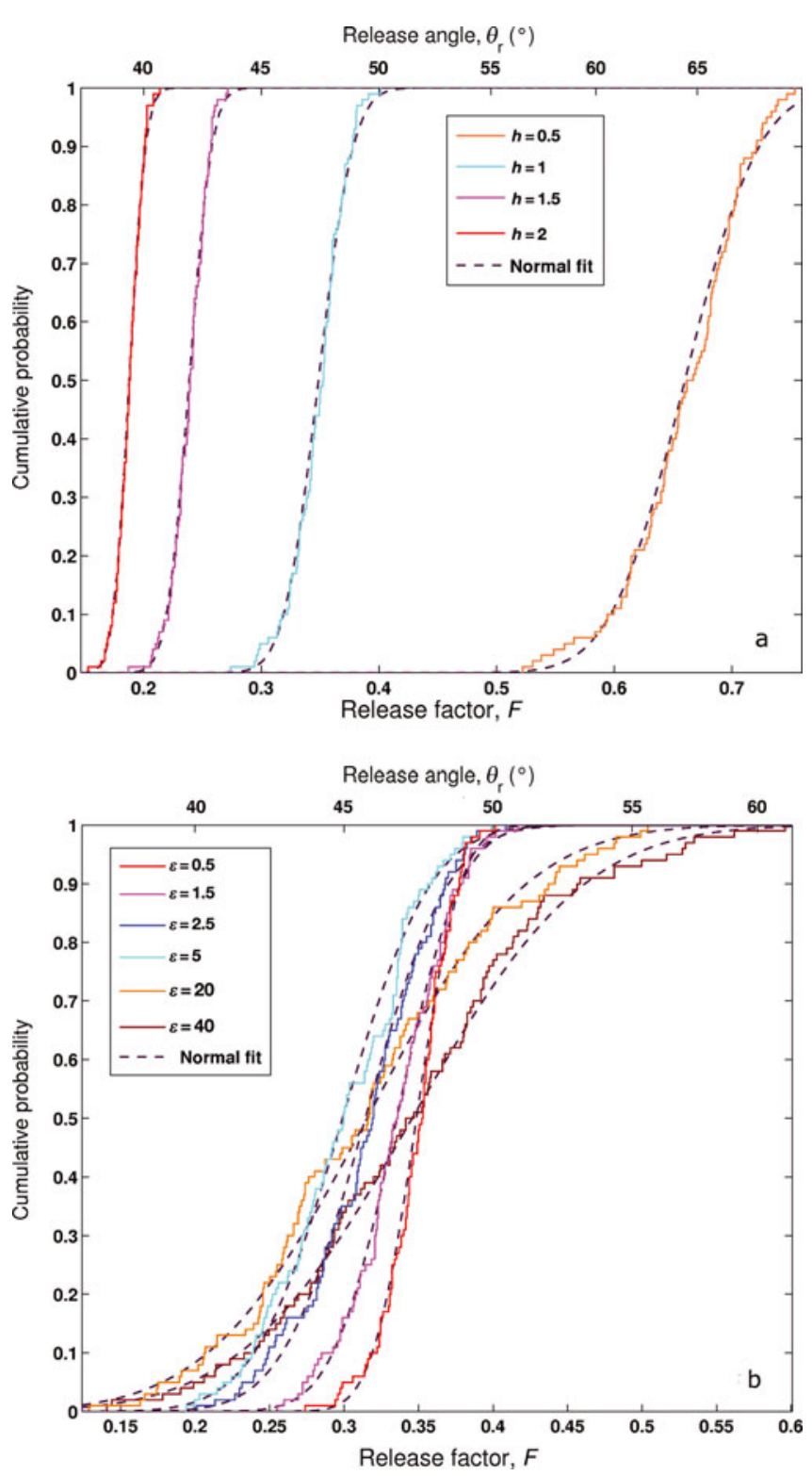

Fig. 10. Cumulative distributions of release angle $\theta_{\mathrm{r}}$ (top scale) and release factor $F$ (bottom scale). (a) $\epsilon=0.5 \mathrm{~m}$ and various values of slab depth, $h$; (b) $h=1 \mathrm{~m}$ and various values of $\epsilon$. Note that according to Eqn (17), the top scale represented is nonlinear in terms of release angle, $\theta_{\mathrm{r}}$. for different values of the slab depth, $h$, varying between 0.25 and $4 \mathrm{~m}$ and different values of the correlation length, $\epsilon$, varying between 0.5 and $40 \mathrm{~m}$. For each couple, $(h, \epsilon), 100$ simulations with different realizations of the heterogeneity were performed. In each of the simulations, the release angle, $\theta_{\mathrm{r}}$, was determined according to the release criterion given by Eqn (5). For reasons that are developed below, the results are primarily presented in terms of the release factor, $F$, defined as

$$
\digamma=\sin \theta_{\mathrm{r}}-\mu \cos \theta_{\mathrm{r}}
$$

\subsection{Release angle and release factor distributions}

Figure 10 shows the influence of slab depth, $h$, and correlation length, $\epsilon$, on the cumulative distributions of the release factor, $F$. Note that these distributions can also be interpreted in terms of the release angle, $\theta_{\mathrm{r}}$. First we observe that all the distributions obtained can be well represented by Gaussian laws, which can be interpreted as a consequence of the Gaussian nature of the cohesion heterogeneity. As shown in Figure 10a, the average and the variance of the release factor distributions decrease with the slab depth, $h$. In addition, the average appears to be approximately independent of the correlation length, $\epsilon$, while the variance increases with $\epsilon$ (Fig. 10b). These results will now be described in more detail.

\subsection{Average release factor}

In a homogeneous case, the release factor, $F_{\mathrm{h}}$, is expected to decrease with $h$ according to $F_{\mathrm{h}}=\langle c\rangle /(\rho g h)$. As shown in Figure 11a, the numerical results appear to closely follow this prediction. In detail, however, it can be noted that the average release factor is always slightly lower than $F_{\mathrm{h}}$, the difference tending to vanish as the slab depth, $h$, increases. The same slight difference with $F_{\mathrm{h}}$ is seen in Figure $11 \mathrm{~b}$, where it appears to increase with increasing correlation length $\epsilon$ for $\epsilon<10 \mathrm{~m}$. Recall that for $\epsilon>10 \mathrm{~m}$ the results begin to be influenced by finite-size effects. These small discrepancies between the results and the theoretical homogeneous value, $F_{\mathrm{h}}$, are due to the heterogeneity and the presence of local cohesion minima. However, globally, we can conclude that the average release factor $\langle F\rangle$ (and the average release angle $\left.\left\langle\theta_{r}\right\rangle\right)$ is almost unaffected by the weaklayer heterogeneity. 

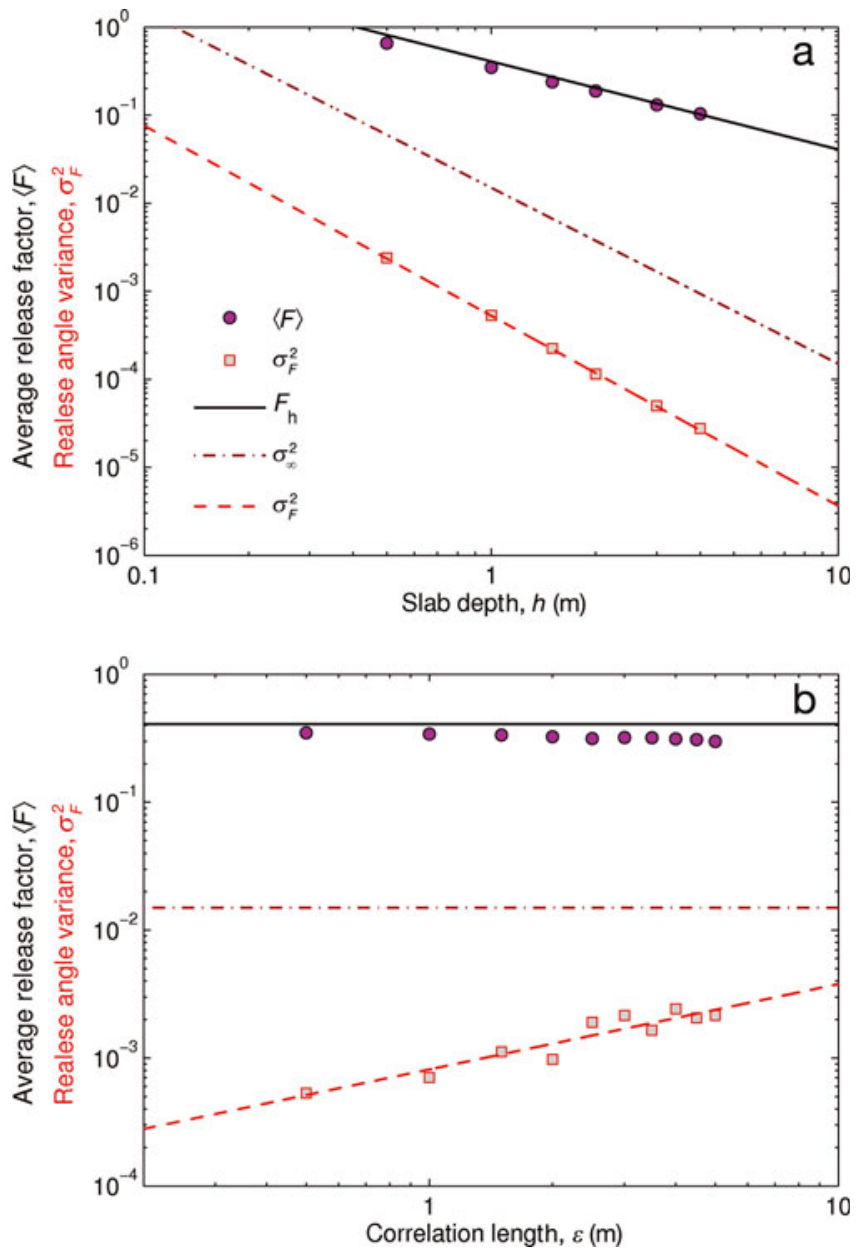

Fig. 11. Evolution of the average release factor, $\langle F\rangle$, and release factor variance, $\sigma_{F}^{2}$, as functions of (a) slab depth, $h$, for $\epsilon=0.5 \mathrm{~m}$ and (b) heterogeneity correlation length, $\epsilon$, for $h=1 \mathrm{~m} . \sigma_{\infty}^{2}$ represents the release factor variance that would be obtained in the case of a completely rigid slab.

\subsection{Variability and heterogeneity smoothing}

Figure 11a shows that the release factor variance decreases with slab depth, $h$, as a power law. The associated exponent is slightly smaller than $-2(\sim-2.16)$. In addition, this variance appears to be significantly smaller than the variance, $\sigma_{\infty}^{2}=\sigma_{c}^{2}(\rho g h)^{-2}$, that would be observed if the stress field in the weak layer exactly followed the heterogeneity variations (the case of a completely rigid slab). This illustrates the smoothing of the heterogeneity due to the elastic redistribution of stresses in the slab.

Following Section 3, the stress redistribution effects induced by slab elasticity are characterized by the smoothing length, $\Lambda=\sqrt{E^{\prime} h u_{\mathrm{p}} / \tau_{\mathrm{p}}}$. Hence we can assume that the ratio $\sigma_{F}^{2} / \sigma_{\infty}^{2}$, can be expressed only in terms of the ratio $\epsilon / \Lambda$. As shown in Figure 12a, all the data points corresponding to $\sigma_{F}^{2} / \sigma_{\infty}^{2}$ obtained from the simulations effectively collapse on a single master curve when plotted in terms of $\epsilon / \Lambda$. The following power-law expression provides a good fit to the data:

$$
\frac{\sigma_{F}^{2}}{\sigma_{\infty}^{2}}=\delta\left(\frac{\epsilon}{\Lambda}\right)^{\frac{2}{3}}
$$

with $\delta=5.45 \times 10^{-2}$. Note, however, that one can expect

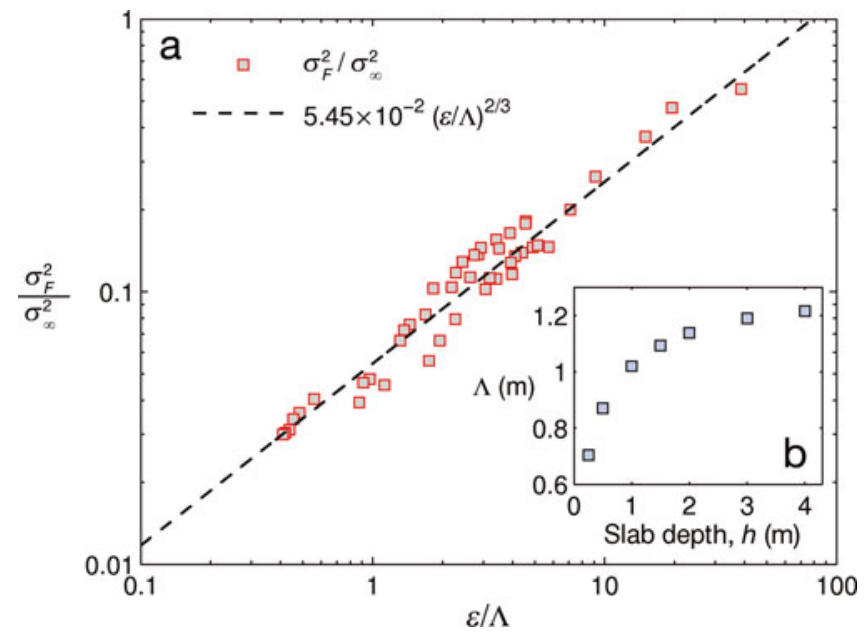

Fig. 12. Ratio between release factor variance, $\sigma_{F}^{2}$, and infinitely rigid slab variance $\sigma_{\infty}^{2}=\sigma_{c}^{2}(\rho g h)^{-2}$, as a function of the ratio between correlation length, $\epsilon$, and elastic smoothing length, $\Lambda$, for all $(h, \epsilon)$ couples ( $\sim 5000$ simulations). (b) Evolution of the elastic smoothing length, $\Lambda$, with slab depth, $h$.

$\sigma_{F}^{2} / \sigma_{\infty}^{2} \rightarrow 1$ if $\epsilon / \Lambda \rightarrow \infty$. Hence, the power law given by Eqn (18) is not expected to remain valid in this limit. In addition, Figure $12 \mathrm{~b}$ shows that the elastic smoothing length, $\Lambda$, slightly increases with slab depth, $h$, in our simulations. This is consistent with the slightly less than -2 power-law exponent observed for the evolution of $\sigma_{F}^{2}$ with $h$ in Figure 11.

We can thus conclude that, at the limit of low values of correlation length, $\epsilon$, and/or high values of the smoothing length, $\Lambda$ (and thus high values of slab depth, $h$ ), $\sigma_{F}^{2} \rightarrow 0$ and the system behaves as in a homogeneous case. This also explains why the difference between the average release factor, $\langle F\rangle$, and the theoretical homogeneous value, $F_{\mathrm{h}}$, decreases when $h$ increases or $\epsilon$ decreases, as noted in Figure $11 \mathrm{a}$ and $\mathrm{b}$. For large correlation lengths, since the effect of $\epsilon$ dominates the effect of $h$ in Eqn (18), very thick slabs can be released even for moderate slope angles, which corresponds to the so-called knock-down effect (Kronholm and Schweizer, 2003; Schweizer and others, 2008).

\section{COMPARISON WITH FIELD DATA}

\subsection{La Plagne release depth data}

La Plagne, in the French Alps, is one of the largest ski areas in the world, covering $100 \mathrm{~km}^{2}$ with $225 \mathrm{~km}$ of ski tracks. Ski patrollers provided us with release depth data from 14391 avalanches collected during the winters of 1998-2010. Since avalanche depths cannot always be directly measured, data come from a mix of visual estimates and precise measurements. From the complete database, 369 naturally released slab avalanches were extracted. These data have been analyzed in detail by Gaume and others (2012). Note that the same data were also used by Failletaz and others (2004), but with fewer avalanches (only three winters). These analyses showed that the release depth cumulative distribution at La Plagne seems to decrease as a power law for large slab depths $(h>0.7 \mathrm{~m}$, corresponding to cumulative exceedance probability lower than $\sim 10 \%$ ). Similar powerlaw trends have also been reported at other locations (Rosenthal and Elder, 2002; McClung, 2003). We note 
however that, due to the error associated with the data, the value of the power-law exponent is poorly constrained and strongly dependent on the cut-off considered for the power law. Typically, in agreement with McClung (2003), exponents in the $[-3 ;-5]$ range provide a good fit to the data.

Our objective here is to examine whether our mechanical model is capable of reproducing these release depth data for both the core and the tail of the distribution. This comparison first requires computing the release depth distribution predicted by the model, which can be obtained in two phases: (1) the release factor distributions presented above have to be inverted to obtain release depth distributions for fixed angle values; (2) these release depth distributions must then be integrated over all slopes, since data mix avalanche paths of various slope angles. Lastly, to be compared with data, the release depth probability obtained from the mechanical model has to be combined with the local snowfall probability.

\subsection{Release depth distributions obtained from mechanical model}

Inversion of release factor distributions

We have shown that the distributions of the release factor, $F$, are normally distributed with mean $\langle F\rangle \approx\langle c\rangle /(\rho g h)$ and variance $\sigma_{F}^{2}=f(\epsilon / \Lambda) \sigma_{c}^{2} /(\rho g h)^{2}$, with $f(\epsilon / \Lambda)$ given by Eqn (18). In addition, since $\Lambda$ varies only slightly in our results (see Fig. 12b), we assume it to be constant in the following. This approximation does not significantly influence the results to be presented, but it allows us to obtain analytical solutions. Hence, the variance, $\sigma_{F}^{2}$, can be written as

$$
\sigma_{F}^{2}=\frac{f(\epsilon) \sigma_{C}^{2}}{(\rho g h)^{2}}
$$

with $f(\epsilon) \approx \kappa \epsilon^{2 / 3}$, and $\kappa=\delta \Lambda^{-2 / 3}$ assumed constant. Finally, the probability density of having a release factor, $F$, for a given slab depth, $h$, is given by

$$
p(F \mid h)=\frac{h}{C_{\sigma} \sqrt{2 \pi}} \mathrm{e}^{-\frac{1}{2}\left(\frac{h F-C_{\mu}}{C_{\sigma}}\right)^{2}},
$$

with $C_{\mu}=\langle c\rangle /(\rho g)$ and $C_{\sigma}=\sigma_{c} \sqrt{f(\epsilon)} /(\rho g)$.

The Mohr-Coulomb rupture criterion, which controls avalanche release in the simulations, can be written in terms of the release factor, $F=\sin \theta_{\mathrm{r}}-\mu \cos \theta_{\mathrm{r}}$, as

$$
\rho g h F=c \text {. }
$$

Hence, slab depth, $h$, and release factor, $F$, play similar roles in this criterion. Consequently, it can be shown that, if the probability density of release factor $F$ for a given slab depth $h$ is $p(F \mid h)=g(F, h)$, then the probability density of $h$ for a given value of $F$ is $p(h \mid F)=g(h, F)$. Equation (20) can thus be inverted to

$$
p(h \mid F)=\frac{F}{C_{\sigma} \sqrt{2 \pi}} \mathrm{e}^{-\frac{1}{2}\left(\frac{h F-C_{\mu}}{C_{\sigma}}\right)^{2}} .
$$

A more detailed and rigorous demonstration of this inversion is provided in Appendix B.

\section{Integration over all slopes}

For the sake of simplicity, we chose, in this study, to consider a uniform probability distribution for the slope factor, $F=\sin \theta-\mu \cos \theta$, between $F_{\min }$ and $F_{\max }: p(F)=1 /$ $\left(F_{\max }-F_{\min }\right)$. Once again, this assumption enables us to obtain analytical expressions for the integrated release depth distribution, $p_{\mathrm{m}}(h)$ :

$$
p_{\mathrm{m}}(h)=\int_{F_{\min }}^{F_{\max }} p(h \mid F) p(F) \mathrm{d} F .
$$

From Eqn (22), we obtain

$$
p_{\mathrm{m}}(h)=\frac{1}{h \sqrt{\pi}}\left[g_{1}(h)+g_{2}(h)\right],
$$

with

$$
\begin{aligned}
g_{1}(h) & =\int_{U_{\min }}^{U_{\max }} u \mathrm{e}^{-u^{2}} \mathrm{~d} u \\
& =\frac{\sqrt{2} C_{\sigma}}{2 h}\left[\mathrm{e}^{-\frac{1}{2} U_{\min }^{2}}-\mathrm{e}^{-\frac{1}{2} U_{\max }^{2}}\right]
\end{aligned}
$$

and

$$
\begin{aligned}
g_{2}(h) & =\int_{U_{\min }}^{U_{\max }} \mathrm{e}^{-u^{2}} \mathrm{~d} u \\
& =\frac{\sqrt{\pi} C_{\mu}}{2 h}\left[\operatorname{Erf}\left(\frac{U_{\min }}{\sqrt{2}}\right)-\operatorname{Erf}\left(\frac{U_{\max }}{\sqrt{2}}\right)\right],
\end{aligned}
$$

where we define $U_{\min }=\left(h F_{\min }-C_{\mu}\right) / C_{\sigma}$ and $U_{\max }=$ $\left(h F_{\max }-C_{\mu}\right) / C_{\sigma}$. In the following, without loss of generality, we assume $F_{\min }=0$ and $F_{\max }=1$.

\subsection{Coupling mechanical and snowfall distributions}

Gaume and others (2012) have shown that the global avalanche release depth probability, $p_{\mathrm{r}}(h)$, resulting from the coupling between the mechanical model presented above and snowfall distributions, can be related to $p_{\mathrm{m}}(h)$ as follows:

$$
\Rightarrow p_{\mathrm{r}}(h) \approx \frac{p_{\mathrm{m}}(h) p_{\mathrm{sf}}\left(h_{\mathrm{sf}} \geq h\right)}{C},
$$

where $p_{\mathrm{sf}}\left(h_{\mathrm{sf}} \geq h\right)$ is the probability of having a snowfall whose thickness, $h_{\mathrm{sf}}$, is higher than the depth, $h$, and $C$ a normalization constant given by $C=\int_{0}^{\infty} p_{\mathrm{m}}(h) p_{\mathrm{sf}}\left(h_{\mathrm{sf}} \geq\right.$ $h) \mathrm{d} h$. This coupling relation expresses that the amount of snowfall represents a limiting factor weighting the mechanical probability density, $p_{\mathrm{m}}(h)$, derived from the stability criterion.

To define the snowfall distribution, $p_{\mathrm{sf}}\left(h_{\mathrm{sf}} \geq h\right)$, Gaume and others (2012) considered the 3 day snowfall annual maxima in La Plagne (MeteoFrance data: daily measurements from 1966) at the average altitude of $2200 \mathrm{~m}$. These maxima follow a generalized extreme value (GEV) distribution so that

$$
p_{\mathrm{sf}}\left(h_{\mathrm{sf}} \geq h\right)=1-\exp \left[-\left(1+\xi_{\mathrm{sf}} \frac{h-\mu_{\mathrm{sf}}}{\sigma_{\mathrm{sf}}}\right)^{-1 / \xi_{\mathrm{sf}}}\right],
$$

where $\mu_{\mathrm{sf}}, \sigma_{\mathrm{sf}}$ and $\xi_{\mathrm{sf}}$ are, respectively, the location, scale and form parameters. These parameters assume the values $\mu_{\mathrm{sf}}=0.98 \mathrm{~m}, \sigma_{\mathrm{sf}}=0.21 \mathrm{~m}$ and $\xi_{\mathrm{sf}}=0.214$.

\subsection{Result of the coupling and sensitivity analysis}

As shown by Gaume and others (2012) (see also Fig. 13), it is possible to find a set of mechanical parameters for which the coupled model described by Eqns (24), (27) and (28) provides a very good adjustment to La Plagne release depth data. The model effectively predicts a power-law behavior of the cumulative exceedence distribution for large slab depths, in good agreement with the empirical distribution, and also accounts well for the data corresponding to lower release depths. In spite of the various assumptions involved, this 


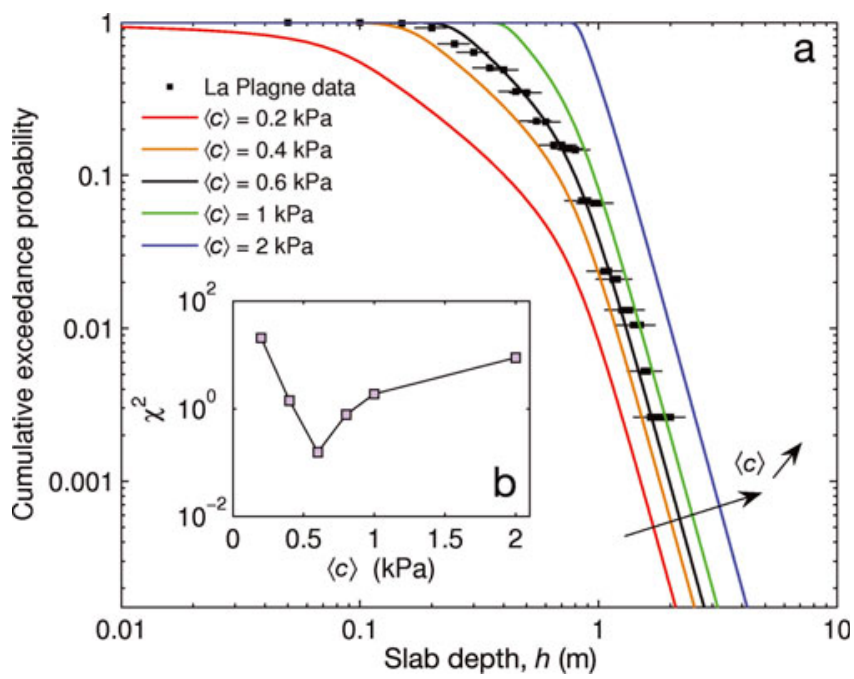

Fig. 13. Slab release depth distributions predicted by the coupled model (Eqn (27)) for different values of average cohesion, $\langle c\rangle$, and comparison with field release depths from La Plagne. The numerical results have been obtained for a cohesion standard deviation $\sigma_{c}=0.3 \mathrm{kPa}$, a correlation length $\epsilon=2 \mathrm{~m}, F_{\min }=0$ and $F_{\max }=1$, the other parameters being the same as in Section 4. (a) Cumulative exceedance probability on logarithmic scale; (b) $\chi^{2}$ of the model $\left(\chi^{2}=\sum\left(p_{\text {data }}-p_{\text {model }}\right)^{2} / p_{\text {model }}\right.$, where $p_{\text {data }}$ and $p_{\text {model }}$ are the cumulative exceedance probabilities derived from the data and from the model, respectively).

model is, to our knowledge, the first capable of reproducing release depth data with such good accuracy. To complement the results shown by Gaume and others (2012), we present below a detailed sensitivity analysis of the predicted distribution of the main mechanical parameters of the model.

Figure 13 shows the comparison between data and the coupled model for different values of the average cohesion, $\langle c\rangle$. The goodness of fit (Fig. 13b) shows a pronounced minimum for $\langle c\rangle=0.6 \mathrm{kPa}$, which indicates that the agreement between model and data strongly depends on this average cohesion value. In particular, the depth, $h_{\mathrm{m}}$, below which no avalanche can occur is strongly dependent on $\langle c\rangle$. This depth can be approximated by (Gaume and others, 2012)

$$
h_{\mathrm{m}} \approx\left(\langle c\rangle-2 \sigma_{c} f(\varepsilon)\right) /\left(\rho g F_{\max }\right) .
$$

With the value $\langle c\rangle=1 \mathrm{kPa}$ retained in Sections 3 and $4, h_{\mathrm{m}}$ is slightly overestimated compared to the data. For $\langle c\rangle=$ $0.6 \mathrm{kPa}$, a value still fully consistent with existing studies (Föhn and others, 1998; Jamieson and Johnston, 2001), an excellent agreement between the coupled model and the data is obtained.

The influence of the cohesion standard deviation, $\sigma_{C}$, is presented in Figure 14. First, it can be noted that $\sigma_{c}$ plays a less significant role than $\langle c\rangle$ in the goodness of the fit. As shown in Figure 14b, values of $\sigma_{c}$ in the 300-500 Pa range give similar results for constant values of the other parameters. In fact, the standard deviation, $\sigma_{C}$, mainly influences the curvature of the coupled cumulative exceedence distribution around the cut-off, $h_{\mathrm{m}}$. Figure $14 \mathrm{a}$ also displays the distribution that would be obtained in the case of a completely rigid slab, which poorly reflects the data. Even if the tail of this distribution could be fitted to the data by tuning the other parameters, there would be no way to properly fit the core of the distribution. This highlights the

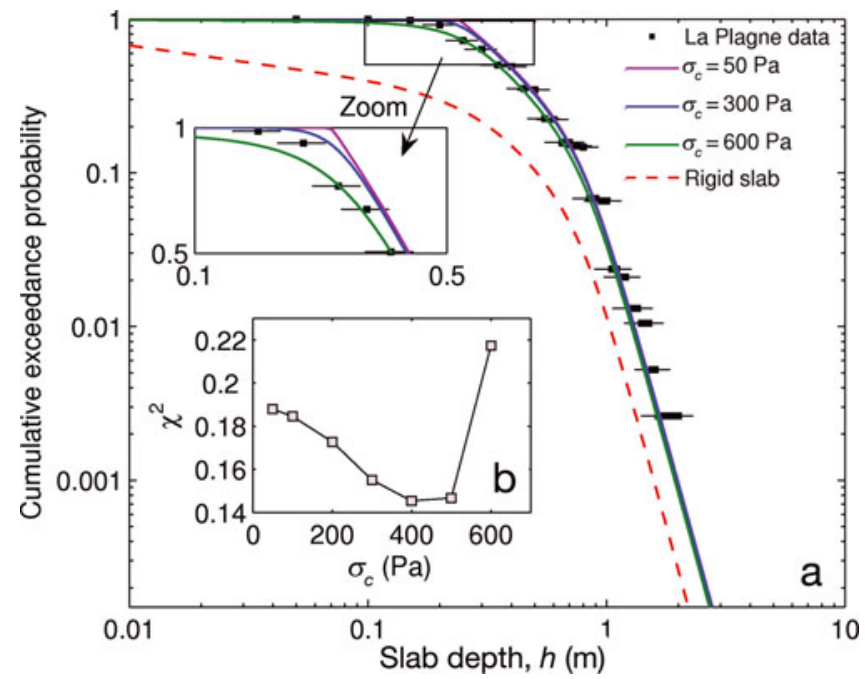

Fig. 14. Slab release depth distributions predicted by the coupled model (Eqn (27)) for different values of the standard deviation, $\sigma_{C}$, and comparison with field release depths from La Plagne. The numerical results have been obtained for a cohesion $\langle c\rangle=0.6 \mathrm{kPa}$, a correlation length $\epsilon=2 \mathrm{~m}, F_{\min }=0$ and $F_{\max }=1$, the other parameters being the same as in Section 4. (a) Cumulative exceedance probability on logarithmic scale; (b) $\chi^{2}$ of the model.

major importance of the elasticity of the slab and of stress redistribution effects.

Finally, the influence of the correlation length, $\epsilon$, is shown in Figure 15. As with the standard deviation, $\sigma_{C}$, the correlation length, $\epsilon$, mainly modifies the curvature of the coupled cumulative exceedence distribution around $h_{\mathrm{m}}$. Globally, the fit to the data remains good for correlation length values in the $0.5-15 \mathrm{~m}$ range.

To conclude, in the range of realistic mechanical parameters for snow (for which stress redistribution effects

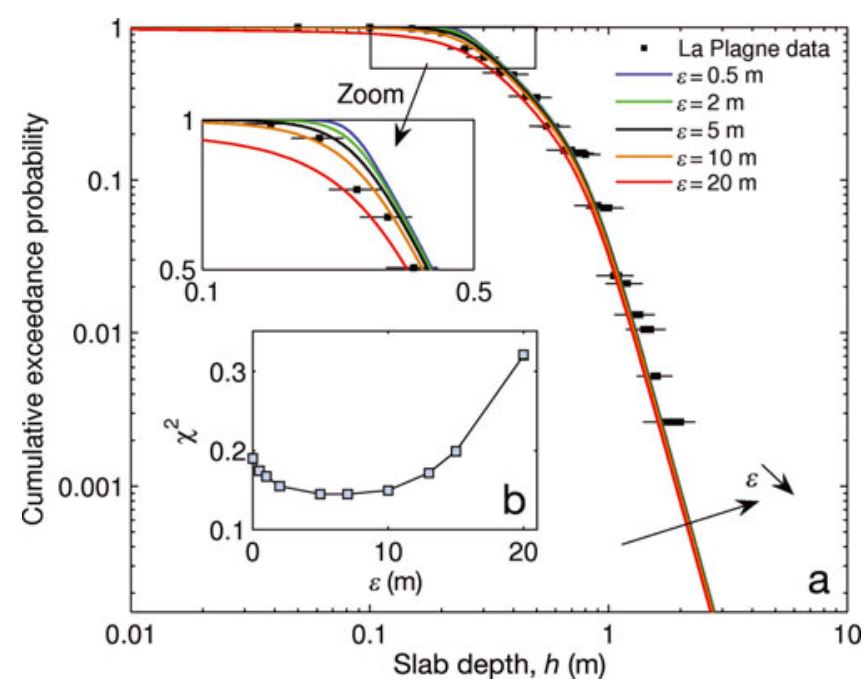

Fig. 15. Slab release depth distributions predicted by the coupled model (Eqn (27)) for different values of the correlation length, $\epsilon$, and comparison with field release depths from La Plagne. The numerical results have been obtained for a cohesion $\langle c\rangle=0.6 \mathrm{kPa}$, a cohesion standard deviation $\sigma_{c}=0.3 \mathrm{kPa}, F_{\min }=0$ and $F_{\max }=1$, the other parameters being the same as in Section 4. (a) Cumulative exceedance probability on logarithmic scale; (b) $\chi^{2}$ of the model. 
play an important role), it turns out that the average cohesion, $\langle c\rangle$, has the most significant influence on slab avalanche release distributions predicted by the coupled model. Hence, provided GEV parameters are known, the adjustment of the model to the data essentially amounts to a one-parameter fit. Note also that the value of $F_{\max }$ which has been set to 1 in the previous results, in fact plays a role essentially similar to that of $\langle c\rangle$ (see Eqn (29)). Hence, changing the value of $F_{\max }$ would result in straightforward modifications of the best-fit value found for $\langle c\rangle$.

\section{CONCLUSION AND PERSPECTIVES}

This paper investigates the influence of weak-layer heterogeneity on slab avalanche release using a finite-element model. A shear-softening interface underlying an elastic slab is modeled and the system is loaded by increasing the slope angle until failure and avalanche release. After validating the model for the case of a nil-cohesion weak spot, the effect of a heterogeneous weak-layer cohesion field was studied. The heterogeneity is represented through a Gaussian distribution, with a spherical covariance function characterized by a spatial correlation length. Release angle distributions were analyzed and a heterogeneity smoothing effect due to redistributions of stresses by elasticity of the slab was highlighted. This smoothing effect induces a reduction of the release angle variance compared to the case of a fully rigid slab. However, the average release angle is almost unaffected by this effect. The presented results show that the smoothing intensity critically depends on the ratio between the correlation length, $\epsilon$, and a characteristic elastic length of the system, $\Lambda$. Further work is required, however, to fully unravel the possible interplay between $\epsilon$ and the cohesion variance, $\sigma_{c}^{2}$, on this smoothing effect. To be compared with field data, the obtained release angle distributions were inverted, yielding a release depth distribution integrated over all slopes. Coupling this mechanical distribution with the distribution of 3 day extreme snowfalls, we were able to reproduce with excellent accuracy field data from 369 natural slab avalanches. A detailed sensitivity analysis showed that this agreement is obtained with only one adjustable parameter, namely the average cohesion, $\langle c\rangle$. The mechanically based probabilistic model thus fulfills the objectives of the study, namely the evaluation of avalanche release depth distributions in any potential release zone, as soon as meteorological data are available. In the future, a straightforward extension to the three-dimensional case will be developed to predict distributions of avalanche release volumes. However, before being used in an operational context, additional tests on other datasets and in other locations need to be performed to further validate the model. Finally, let us recall that, with the parameters used in our model, the crown fracture always occurs at particular morphological features (e.g. ridges, rocks and trees) since the heterogeneity is not sufficient to directly trigger tensile rupture within the slab. Thus, another interesting perspective for future work is to study the release depth distributions obtained with different sets of parameters leading to other types of failure mechanisms.

\section{ACKNOWLEDGEMENTS}

This work was supported by the European DYNAVAL and $M P^{3}$ INTERREG projects and the French ANR MOPERA project. We thank Claude Schneider, snow scientist in La Plagne, for providing release depth data and MeteoFrance for providing related snowfall data. An anonymous reviewer and the Scientific Editor P. Bartelt are gratefully acknowledged for their careful reading of the manuscript and their constructive comments.

\section{REFERENCES}

Bader HP and Salm B (1990) On the mechanics of snow slab release. Cold Reg. Sci. Technol., 17(3), 287-300 (doi: 10.1016/ S0165-232X(05)80007-2)

Barbolini M, Gruber U, Keylock CJ, Naaim M and Savi F (2000) Application of statistical and hydraulic-continuum densesnow avalanche models to 5 real European sites. Cold Reg. Sci. Technol., 31(2), 133-149 (doi: 10.1016/S0165-232X(00) 00008-2)

Bartelt P, Salm B and Gruber U (1999) Calculating dense-snow avalanche runout using a Voellmy-fluid model with active/ passive longitudinal straining. J. Glaciol., 45(150), 242-254

Bažant ZP, Zi G and McClung D (2003) Size effect law and fracture mechanics of the triggering of dry snow slab avalanches. J. Geophys. Res., 108(B2), 2119 (doi: 10.1029/2002JB001884)

Bellaire S and Schweizer J (2011) Measuring spatial variations of weak layer and slab properties with regard to snow slope stability. Cold Reg. Sci. Technol., 65(2), 234-241 (doi: 10.1016/ j.coldregions.2010.08.013)

Birkeland K, Kronholm K and Logan S (2004) A comparison of the spatial structure of the penetration resistance of snow layers in two different snow climates. In Ganju A ed. Proceedings of the International Symposium on Snow Monitoring and Avalanches, 12-16 April 2004, Manali, India. Snow and Avalanche Study Establishment, Manali, 3-11

Camponovo C and Schweizer J (2001) Rheological measurements of the viscoelastic properties of snow. Ann Glaciol., 32, $44-50$

Charras T and Di Paola F (2011) Utiliser Cast3M: la procédure PASAPAS. Direction de I'Énergie Nucléaire du Commissariat à I'Énergie Atomique et aux Énergies Alternatives (CEA), Paris http:// www-cast3m.cea.fr/html/Documentation_Cast3M/Pasapas.pdf

Chiaia BM, Cornetti P and Frigo B (2008) Triggering of dry snow slab avalanches: stress versus fracture mechanical approach. Cold Reg. Sci. Technol., 53(2), 170-178 (doi: 10.1016/j.coldregions.2007.08.003)

Chilès JP and Delfiner P (1999) Geostatistics: modeling spatial uncertainty. Wiley, Hoboken, NJ

Conway H and Abrahamson J (1988) Snow-slope stability - a probabilistic approach. J. Glaciol., 34(117), 170-177

De Montmollin V (1978) Introduction à la rhéologie de la neige. (PhD thesis, Université Scientifique et Médicale de Grenoble)

Eckert N, Parent E, Naaim M and Richard D (2008) Bayesian stochastic modelling for avalanche predetermination: from a general system framework to return period computations. Stoch. Environ. Res. Risk Assess., 22(2), 185-206 (doi: 10.1007/ s00477-007-0107-4)

Eckert N, Naaim M and Parent E (2010) Long-term avalanche hazard assessment with a Bayesian depth-averaged propagation model. J. Glaciol., 56(198), 563-586 (doi: 10.3189/ 002214310793146331)

Faillettaz J, Louchet F and Grasso JR (2004) Two-threshold model for scaling laws on noninteracting snow avalanches. Phys. Rev. Lett., 93(20), 208001 (doi: 10.1103/PhysRevLett.93.208001)

Föhn PMB, Camponovo C and Krüsi G (1998) Mechanical and structural properties of weak snow layers measured in situ. Ann. Glaciol., 26, 1-6

Fyffe B and Zaiser M (2004) The effects of snow variability on slab avalanche release. Cold Reg. Sci. Technol., 40(3), 229-242 (doi: 10.1016/j.coldregions.2004.08.004) 
Fyffe B and Zaiser M (2007) Interplay of basal shear fracture and slab rupture in slab avalanche release. Cold Reg. Sci. Technol., 49(1), 26-38 (doi: 10.1016/j.coldregions.2006.09.011)

Gaume J, Chambon G, Naaim M and Eckert N (2011) Influence of the weak layer's heterogeneity on slab avalanches releases using a finite element method. In Bonelli S, Dascalu C and Nicot F eds. Advances in Bifurcation and Degradation in Geomaterials: Proceedings of the 9th International Workshop on Bifurcation and Degradation in Geomaterials, 23-26 May 2011, Porquerolles Island, Provence, France. Springer, Dordrecht, 261-266

Gaume J, Chambon G, Eckert N and Naaim M (2012) Relative influence of mechanical and meteorological factors on avalanche release depth distributions. Geophys. Res. Lett., 39(12), L12401 (doi: 10.1029/2012GL051917)

Griffiths DV and Fenton G (2004) Probabilistic slope stability analysis by finite elements. J. Geotech. Geoenviron. Eng., 130(5), 507-518 (doi: 10.1061/(ASCE)1090-0241(2004)130:5(507))

Heierli J and Zaiser M (2008) Failure initiation in snow stratifications containing weak layers: nucleation of whumpfs and slab avalanches. Cold Reg. Sci. Technol., 52(3), 385-400 (doi: 10.1016/j.coldregions.2007.02.007)

Heierli J, Gumbsch P and Zaiser M (2008) Anticrack nucleation as triggering mechanism for snow slab avalanches. Science, 321(5886), 240-243 (doi: 10.1126/science.1153948)

Hutter K (1996) Avalanche dynamics. In Singh VP ed. Hydrology of disasters. Kluwer Academic Publishers, Dordrecht, 317-394

Jamieson JB and Johnston CD (1990) In-situ tensile tests of snowpack layers. J. Glaciol., 36(122), 102-106

Jamieson B and Johnston CD (2001) Evaluation of the shear frame test for weak snowpack layers. Ann. Glaciol., 32, 59-69 (doi: 10.3189/172756401781819472)

Jamieson JB and Schweizer J (2000) Texture and strength changes of buried surface-hoar layers with implications for dry snow-slab avalanche release. J. Glaciol., 46(152), 151-160 (doi: 10.3189/ 172756500781833278)

Jamieson B, Margreth S and Jones A (2008) Application and limitations of dynamic models for snow avalanche hazard mapping. In Campbell C, Conger S and Haegeli $\mathrm{P}$ eds. Proceedings of the 14th International Snow Science Workshop, 21-27 September 2008, Whistler, BC, Canada. International Snow Science Workshop, Whistler, BC, 730-739

Johnson BC, Jamieson B and Stewart R (2004) Seismic measurement of fracture speed in a weak snowpack layer. Cold Reg. Sci. Technol., 40(1-2), 41-45 (doi: 10.1016/j.coldregions.2004. 05.003)

Keylock CJ, McClung DM and Magnísson MM (1999) Avalanche risk mapping by simulation. J. Glaciol., 45(150), 303-314

Kronholm K (2004) Spatial variability of snow mechanical properties with regard to avalanche formation. (PhD thesis, University of Zürich)

Kronholm K and Birkeland KW (2005) Integrating spatial patterns into a snow avalanche cellular automata model. Geophys. Res. Lett., 32(19), L19504 (doi: 10.1029/2005GL024373)

Kronholm K and Schweizer J (2003) Snow stability variation on small slopes. Cold Reg. Sci. Technol., 37(3), 453-465 (doi: 10.1016/S0165-232X(03)00084-3)

Mahajan P and Joshi SK (2008) Modeling of interfacial crack velocities in snow. Cold Reg. Sci. Technol., 51(2-3), 98-111 (doi: 10.1016/j.coldregions.2007.05.008)

Mahajan P, Kalakuntla R and Chandel C (2010) Numerical simulation of failure in a layered thin snowpack under skier load. Ann. Glaciol., 51(54), 169-175 (doi: 10.3189/ 172756410791386436)

McClung DM (1977) Direct simple shear tests on snow and their relation to slab avalanche formation. J. Glaciol., 19(81), 101-109

McClung DM (1979) Shear fracture precipitated by strain softening as a mechanism of dry slab avalanche release. J. Geophys. Res., 84(B7), 3519-3526 (doi: 10.1029/JB084iB07p03519)

McClung D (2003) Size scaling for dry snow slab release. J. Geophys. Res., 108(B10), 2465 (doi: 10.1029/2002JB002298)
McClung DM (2009) Dry snow slab quasi-brittle fracture initiation and verification from field tests. J. Geophys. Res., 114(F1), F01022 (doi: 10.1029/2007JF000913)

McClung DM (2011) Analysis of critical length measurements for dry snow slab weak-layer shear fracture. J. Glaciol., 57(203), 557-566 (doi: 10.3189/002214311796905541)

McClung DM and Schweizer J (2006) Fracture toughness of dry snow slab avalanches from field measurements. J. Geophys. Res., 111(F4), F04008 (doi: 10.1029/2005JF000403)

Mellor M (1975) A review of basic snow mechanics. IAHS Publ. 114 (Symposium at Grindelwald 1974 - Snow Mechanics), 251-291

Meunier M and Ancey C (2004) Towards a conceptual approach to predetermining long-return-period avalanche run-out distances. J. Glaciol., 50(169), 268-278 (doi: 10.3189/ 172756504781830178)

Naaim M, Faug T and Naaim-Bouvet F (2003) Dry granular flow modelling including erosion and deposition. Surv. Geophys., 24(5-6), 569-585 (doi: 10.1023/B:GEOP.0000006083. $47240.4 \mathrm{c})$

Narita H (1980) Mechanical behaviour and structure of snow under uniaxial tensile stress. J. Glaciol., 26(94), 275-282

Navarre JP, Taillefer A and Danielou Y (1992) Fluage et rhéologie de la neige. In Brugnot $\mathrm{G}$, Brun E, Gubler H, Norem $\mathrm{H}$ and Reynaud L eds. Symposium de Chamonix CISA-IKAR. Les apports de la recherche scientifique à la neige, glace et avalanches, 4-8 Juin 1991, Chamonix, France. Actes du Symposium. Association Nationale pour l'Étude de la Neige et des Avalanches (ANENA), Grenoble, 377-388

Palmer AC and Rice JR (1973) The growth of slip surfaces in the progressive failure of over-consolidated clay. Proc. $R$. Soc. London, Ser. A, 332(1591), 527-548 (doi: 10.1098/ rspa.1973.0040)

Reiweger I, Schweizer J, Dual J and Herrmann HJ (2009) Modelling snow failure with a fibre bundle model. J. Glaciol., 55(194), 997-1002

Roch A (1966) Les variations de la résistance de la neige. IAHS Publ. 69 (Symposium at Davos 1965 - Scientific Aspects of Snow and Ice Avalanches), 86-99

Rosenthal W and Elder K (2002) Evidence of chaos in slab avalanches. In Stevens JR ed. Proceedings of the 11th International Snow Science Workshop, 29 September-4 October 2002, Penticton, British Columbia. Snow Avalanche Programs, BC Ministry of Transportation, Victoria, BC, 243-253

Schweizer J (1998) Laboratory experiments on shear failure of snow. Ann. Glaciol., 26, 97-102

Schweizer J (1999) Review of dry snow slab avalanche release. Cold Reg. Sci. Technol., 30(1-3), 43-57 (doi: 10.1016/S0165232X(99)00025-7)

Schweizer J, Jamieson JB and Schneebeli M (2003) Snow avalanche formation. Rev. Geophys., 41(4), 1016 (doi: 10.1029/ 2002RG000123)

Schweizer J, Kronholm K, Jamieson JB and Birkeland KW (2008) Review of spatial variability of snowpack properties and its importance for avalanche formation. Cold Reg. Sci. Technol., 51(2-3), 253-272 (doi: 10.1016/j.coldregions.2007.04.009)

Sigrist C (2006) Measurement of fracture mechanical properties of snow and application to dry snow slab avalanche release. (PhD thesis, ETH Zürich)

Stoffel M (2005) Numerical modelling of snow using finite elements. (PhD thesis, ETH Zürich)

Van Herwijnen A and Heierli J (2009) Measurement of crack-face friction in collapsed weak snow layers. Geophys. Res. Lett., 36(23), L23502 (doi: 10.1029/2009GL040389)

Verpeaux P, Charras T and Millard A (1988) CASTEM 2000: une approche moderne du calcul des structures. In Fouet J-M, Ladevèze $\mathrm{P}$ and Ohayon $\mathrm{R}$ eds. Calcul des structures et intelligence artificielle, Vol. 2. (Collection méthodes numériques dans les sciences de l'ingénieur) Pluralis, Saint Etienne, 261-271 http://www-cast3m.cea.fr/ 


\section{APPENDIX A. MODEL VALIDATION: EXPRESSION OF THE PARAMETERS}

We give here the expressions of the parameters involved in the weak-spot analytical solution derived in Section 3.1:

$$
\begin{gathered}
r=\frac{\frac{\Lambda}{\Lambda_{\mathrm{ws}}}\left(1-\frac{\Lambda_{\mathrm{ws}}^{2}}{\Lambda^{2}}\right)\left(\beta \mathrm{e}^{a / \Lambda_{\mathrm{ws}}^{2}}-\alpha \mathrm{e}^{-a / \Lambda_{\mathrm{ws}}^{2}}\right)}{1+\frac{\Lambda}{\Lambda_{\mathrm{ws}}}\left(\beta \mathrm{e}^{a / \Lambda_{\mathrm{ws}}^{2}}-\alpha \mathrm{e}^{-a / \Lambda_{\mathrm{ws}}^{2}}\right)}, \\
\alpha=\frac{1-\mathrm{e}^{-a / \Lambda_{\mathrm{ws}}}}{\mathrm{e}^{\mathrm{a} / \Lambda_{\mathrm{ws}}}-\mathrm{e}^{-2 a / \Lambda_{\mathrm{ws}}},} \\
\beta=\frac{1-\alpha \mathrm{e}^{-a / \Lambda_{\mathrm{ws}}}}{\mathrm{e}^{a / \Lambda_{\mathrm{ws}}}}, \\
r^{\prime}=\frac{\Lambda^{2}}{\Lambda_{\mathrm{ws}}^{2}}-1-r, \\
r_{2}=\frac{a}{\Lambda}\left(\frac{\tau_{\mathrm{r}}}{\tau_{\mathrm{g}}}-1\right) .
\end{gathered}
$$

\section{APPENDIX B. INVERSION OF RELEASE FACTOR DISTRIBUTIONS}

The objective is to deduce from the distributions, $p(F \mid h)$, derived from the simulations, the distributions, $p(h \mid F)$, that would be obtained in the 'dual' experiment (much more difficult to perform numerically) consisting of fixing the slope angle and gradually increasing the slab depth, $h$, until rupture. The principle of this inversion lies in that, for a given realization of heterogeneity, the rupture is achieved under the same conditions in both experiments. Hence, the couple $(F, h)$ obtained in both cases must be the same.

It is thus possible to obtain $p(h \mid F)$ from $p(F \mid h)$ by generating a large number of couples $(F, h)$ in drawing in $p(F \mid h)$ distributions for several values of $h$, and then to reclassify data obtained as a function of $F$. To this end, values of $h$ can be drawn from a random distribution, $p(h)$. Instead of applying this protocol empirically, one can notice that the knowledge of $p(h)$ allows us to consider the couple $(F, h)$ as a random vector, and to compute $p(h \mid F)$ using Bayes's formula:

$$
p(h \mid F)=\frac{p(F \mid h) p(h)}{\int_{-\infty}^{+\infty} p(F \mid h) p(h) \mathrm{d} h} .
$$

Then, to avoid biasing the result, it is necessary to sample uniformly all possible values of $h$. In other words, $p(h)$ has to be chosen as constant. Equation (B1) therefore simplifies to

$$
p(h \mid F)=\frac{p(F \mid h)}{\int_{-\infty}^{+\infty} p(F \mid h) \mathrm{d} h} .
$$

Knowing the expression for $p(F \mid h)$

$$
p(F \mid h)=\frac{1}{\sqrt{2 \pi} \sigma_{F}} \mathrm{e}^{-\frac{1}{2}\left(\frac{F-\langle F\rangle}{\sigma_{F}}\right)^{2}},
$$

with

$$
\langle F\rangle=\frac{\langle c\rangle}{\rho g h}
$$

and

$$
\sigma_{F}=\frac{\sigma_{C}}{\rho g h} \sqrt{f\left(\frac{\epsilon}{\Lambda}\right)}
$$
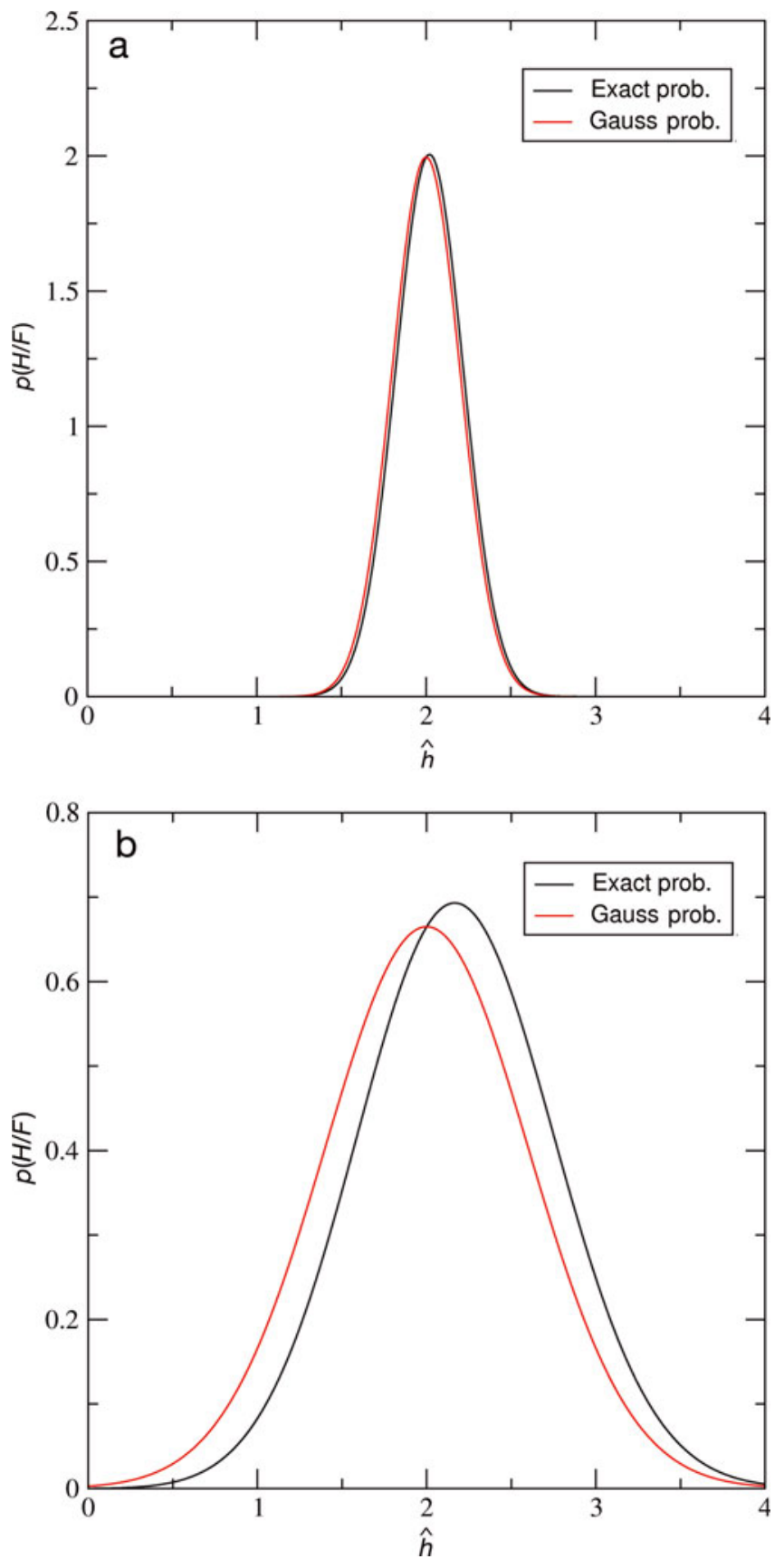

Fig. 16. Comparison between the exact expression (Eqn (B7)) and the Gaussian approximate expression (Eqn (B8)) of the inverted probability, $p(\widehat{h} \mid F)$ (case $F=0.5$ ): (a) $C_{V}=0.1$; (b) $C_{V}=0.3$.

where $\Lambda$ is the elastic characteristic length of the system, the inverse distribution, $p(h \mid F)$, can be obtained numerically by applying Eqn (B2). In the present case, in which $p(F \mid h)$ is Gaussian, it is possible to analytically integrate the denominator, assuming that $\Lambda=\Lambda_{0}$ is a constant independent of $h$. As already mentioned, this assumption is not completely fulfilled, but the error made is negligible, since the influence of $h$ on the function $\sigma_{F} \rho g h / \sigma_{C}=\sqrt{f(\epsilon / \Lambda)}$ remains low.

For homogeneity reasons, we also define $\widehat{h}=\rho g h /\langle c\rangle$ (dimensionless variable). We can then write

$$
p(F \mid \widehat{h})=\frac{\widehat{h}}{\sqrt{2 \pi} C_{V}} \mathrm{e}^{-\frac{1}{2}\left(\frac{\widehat{h F F-1}}{C_{V}}\right)^{2}},
$$


with $C_{V}=\left(\sigma_{c} /\langle c\rangle\right) \sqrt{f\left(\epsilon / \Lambda_{0}\right)}$. We thus obtain

$$
\begin{aligned}
\int_{-\infty}^{+\infty} p(F \mid \widehat{h}) \mathrm{d} \widehat{h} & =\frac{1}{\sqrt{2} \pi C_{V}} \int_{-\infty}^{+\infty} \widehat{h} \mathrm{e}^{-\frac{1}{2}\left(\frac{\hat{h F-1}}{C_{V}}\right)^{2} \widehat{d h}} \\
& =\frac{1}{F^{2}}
\end{aligned}
$$

Finally, from Eqn (B2):

$$
p(\widehat{h} \mid F)=\frac{F^{2} \widehat{h}}{\sqrt{2 \pi} C_{V}} \mathrm{e}^{-\frac{1}{2}\left(\frac{\widehat{h F-1}}{C_{V}}\right)^{2}} .
$$

Hence, strictly, the inverted probability distribution, $p(\widehat{h} \mid F)$, is not Gaussian. However, as shown in Figure 16, if the Gaussian contribution to Eqn (B7) is sufficiently sharp, i.e. if $C_{V}$ is sufficiently small, the variation of $\widehat{h}$ in the prefactor remains negligible. We can then replace $\widehat{h}$ in the prefactor by the mode of the Gaussian, i.e. $1 / F$, which leads to the following approximate expression:

$$
p(\widehat{h} \mid F)=\frac{F}{\sqrt{2 \pi} C_{V}} \mathrm{e}^{-\frac{1}{2}\left(\frac{\widehat{h F-1}}{C_{V}}\right)^{2}},
$$

which is a Gaussian. This approximation is well justified in our case, since for $\epsilon<10 \mathrm{~m}$ we have $C_{V}<10 \%$ (Fig. 16a). Note that in the case of a completely rigid slab, the coefficient of variation, $C_{\mathrm{V}}$, would be equal to that of the cohesion, $\sigma_{c} /\langle c\rangle=30 \%$, a value for which the Gaussian approximation is less valid (Fig. 16b). The role of the variance reduction by elastic effects (i.e. the role of the function $f(\epsilon / \Lambda)$ ) is thus crucial for this Gaussian approximation of $p(h \mid F)$ to be valid. Returning to the physical variable $h$, we finally obtain Eqn (22), which simply corresponds to the expression of $p(F \mid h)$ in which the variables $F$ and $h$ have been inverted (again under the assumption that $\Lambda=\Lambda_{0}=$ constant). 